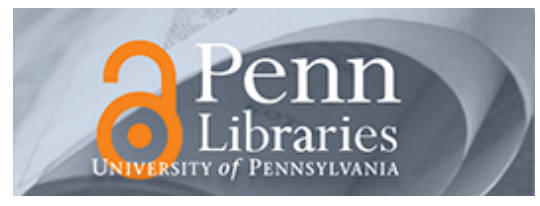

University of Pennsylvania

ScholarlyCommons

Accounting Papers

Wharton Faculty Research

4-2011

\title{
Soft Information and the Stewardship Value of Accounting \\ Disclosure
}

Mirko S. Heinle

University of Pennsylvania

Christian Hofmann

Follow this and additional works at: https://repository.upenn.edu/accounting_papers

Part of the Accounting Commons, and the Corporate Finance Commons

Recommended Citation

Heinle, M. S., \& Hofmann, C. (2011). Soft Information and the Stewardship Value of Accounting

Disclosure. OR Spectrum, 33 (2), 333-358. http://dx.doi.org/10.1007/s00291-010-0215-2

This paper is posted at ScholarlyCommons. https://repository.upenn.edu/accounting_papers/84

For more information, please contact repository@pobox.upenn.edu. 


\title{
Soft Information and the Stewardship Value of Accounting Disclosure
}

\author{
Abstract \\ ongoing debate about increasing the disclosure of soft information, we investigate the economic \\ include market price as a performance measure and investigate whether the principal benefits from \\ result follows from either a noisier or a less congruent market price as a consequence of disclosing \\ costs or the uncertainty of information endowment.

\section{Keywords} \\ managerial incentives, stock-based compensation, financial reporting, disclosure \\ Disciplines \\ Accounting | Corporate Finance
}

In light of IASB's statement to drop stewardship as a separate objective of financial accounting and the consequences of publicly reported soft information from a stewardship perspective. In an LEN model we disclosing additional information. While the principal can only use contractible performance measures in the contract with the agent, capital market participants can only use disclosed information when pricing firm value. We find that the disclosure of information can decrease the principal's expected net profit. This additional information. Thus, we present a rationale for partial disclosure in the absence of proprietary 


\title{
SOFT INFORMATION AND THE STEWARDSHIP VAlue of AcCounting Disclosure*
}

by

\author{
Mirko S. Heinle \\ University of Mannheim \\ mirko.heinle@bwl.uni-mannheim.de \\ Christian Hofmann \\ University of Mannheim \\ hofmann@bwl.uni-mannheim.de
}

January 28, 2009

\begin{abstract}
:
In the light of IASB's statement to drop stewardship as a separate objective of financial accounting and the ongoing debate about increasing the disclosure of soft information, we investigate the economic consequences of publicly reported soft information from a stewardship perspective. In a LEN model we include market price as a performance measure and investigate whether the principal benefits from disclosing additional information. While the principal can only use contractible performance measures in the contract with the agent, capital market participants can only use disclosed information when pricing firm value. We find that the disclosure of information can decrease the principal's expected net profit. This result follows from either a noisier or a less congruent market price as a consequence of disclosing additional information. Thus, we present a rationale for partial disclosure in the absence of proprietary costs or the uncertainty of information endowment.
\end{abstract}

\footnotetext{
* We thank Stan Baiman, Robert Göx, Thomas Pfeiffer, Stefan Reichelstein and participants at research seminars at the Annual Meeting of the German Academic Association for Business Research (VHB), the IV. Summer School on Accounting 2008, and at the Wharton School. Christian Hofmann gratefully acknowledges financial support for this project from the Deutsche Forschungsgemeinschaft (HO 2430/3-1).
} 


\section{INTRODUCTION}

Standard setters and regulators justify reporting and disclosure requirements by the demand of capital owners for decision-useful information (O'Connell 2007, 217). Following the argumentation applied by FASB and IASB, a greater volume of timely information enhances market efficiency and decreases the cost of capital. More recently, regulators require firms to increase the disclosure of soft information. ${ }^{1}$ Soft information "includes less certain information known to an issuer, such as projections and other forward-looking information" (SEC Commissioner R.Y. Roberts 1994). More generally, we define soft information as information that cannot be verified and, thus, cannot be used in a contract between two parties (i.e., the information is non-contractible). ${ }^{2}$ This definition covers such diverse examples as information on sales forecasts, the progress of an investment project (Choi, Kristiansen and Nahm 2007), management reputation (Stocken and Verrecchia 1999), and the closeness of the relation between a firm and its bank (Liberti 2003).

We argue that, while disclosing information to investors may improve market price informativeness, it can be detrimental to firm owners from a stewardship perspective. More specifically, we identify conditions under which the value of publicly reported information is negative. Key to the negative value is the dilution of managerial incentives as a consequence of a noisier and less congruent market price that results when the disclosed information is impounded in the price. Therefore, gains in price informativeness come at the expense of firm value.

Disclosure of soft information is pervasive. For example, Tsao (2002) notes that “a company's own filings to the Securities \& Exchange Commission are filled with revealing information about prospects for sales and profits." Empirical evidence suggests that soft information in earnings announcements is value relevant (Davis, Piger and Sedor 2007) and affects price volatility (Demers and Vega 2008). Besides firm disclosures, analysts' reports and press-related statements release further soft information to investors

\footnotetext{
${ }^{1}$ Examples refer to the introduction of the "soft harbor rule" in 1979 and its reform in 1995, which both tried to encourage the disclosure of forward looking information, Seamons and Rouse (1997).

${ }^{2}$ Similarly, Petersen $(2004,2)$ defines soft information as "information which is difficult to completely summarize in a numeric score."
} 
(Tetlock, Saar-Tsechansky and Macskassy 2008).

Understanding the stewardship value of accounting disclosures is important because regulators frequently presume complementarity between stewardship and decision-usefulness. For example, the IASB recently announced that “... the Board agreed that stewardship or accountability should not be a separate objective of financial reporting by business entities in the converged framework" (IASB 2005, para. 24). More specifically, the IASB claims that "financial information directed at the primary objective of providing information useful for investment [...] is useful for other purposes, including assessing management's stewardship" (IASB 2005, para. 24). Bushman, Engel and Smith (2006) provide supportive evidence for this claim. On the contrary, theoretical analysis by Gjesdal (1981), Paul (1992), and Feltham and Xie (1994) emphasizes potential conflicts between stewardship and decision-usefulness; e.g., Gassen (2008) provides supportive evidence for this view. By identifying conditions where extensive mandatory disclosure of soft information destroys firm value, our study yields important insights to the regulator.

Given that market price aggregates any information available to investors, by using market price as a performance measure in the contract with the manager, firm owners are able to indirectly contract on otherwise non-contractible information (Bushman and Indjejikian 1993). ${ }^{3}$ In situations where the accounting system internally generates soft information (e.g., a report that compiles "soft facts" that may be important to investment decisions or projections that are necessary when preparing financial statements), this result suggests that it can be beneficial for firm owners to publicly report this soft information (e.g., by including the information in the management report). However, it is unclear at this point whether firm owners should disclose the entire amount or merely a subset of the soft information. In this regard, our results shed light on

\footnotetext{
3 This statement hinges on the contracting usefulness of soft information. Whereas, e.g., information on the success of an in-house training program or the success of implementing a new software are straightforward examples of useful signals, forwardlooking information can be useful as well. Even though participants in an agency would most likely not want to sign a contract on pure expectations, prospective information often contains information about past performance. For example,

Information that may be informative about the manager's effort, but that may not be contractible, includes information about the relative performance of the firm within the industry and information about the manager's reputation. (Stocken and Verrecchia 1999)

Likewise,

... analysts can help interpret the implication of a newly announced technology development for future performance; and corporate disclosures can provide facts and explanations to confirm or deny speculations, predictions, and forecasts made by analysts. (Chen, Cheng and Lo 2006, 2)
} 
optimal firm-level disclosure policy.

This study first contributes to the discussion on the economic consequences of mandatory disclosure. In particular, we address the disclosure of soft information and identify the incremental stewardship value of varying the quantity of publicly reported information. By identifying conditions under which a larger quantity of disclosed information yields a negative value, we establish that increasing mandatory disclosure of soft information can decrease firm value. Secondly, we present a rationale for the firm not to follow a fulldisclosure strategy but rather to commit ex ante to withhold some information from capital market participants. More specifically, given that soft information is available to investors from other sources outside the firm, there exist conditions where it is beneficial to not disclose supplemental (hard or soft) information. In this respect, the study contributes to the discussion on voluntary disclosure. The study also contributes to the ongoing discussion on the relation of stewardship and decision-usefulness. In particular, our results support the opponents of the IASB's proposal to drop stewardship as a separate objective of financial accounting.

In our analysis, an agent performs multiple tasks and is evaluated using multiple performance measures. While we only consider performance measures that are (conditionally) informative about the agent's actions, only a subset of these signals can be used by the principal in the contract with the agent (i.e., only a subset of the generated signals is contractible information). In addition, a subset of the generated signals is publicly reported to capital market participants ${ }^{4}$ who use all information available (including non-contractible information) to form expectations about firm value. In the model, the gross market price is equal to investors' expectation about the firm's terminal payoff conditional on the available information and their beliefs about the actions selected by the agent. To simplify the analysis, we assume the principal is risk neutral, the agent has exponential utility and quadratic effort cost, the performance measures are normally distributed, and the compensation contract is restricted to be a linear function of the contractible performance measures, including

\footnotetext{
${ }^{4}$ Hayes and Schaefer (2000) provide empirical evidence that supports the hypothesis that firms' contracts include performance measures that are only observable by the contracting parties and that these measures are informative about the firm's future prospects.
} 
market price. ${ }^{5}$ We determine the value of publicly reported (supplemental) information by comparing the expected net payoff to the principal under two information systems; the information systems differ regarding the disclosure of a single performance measure. This disclosure value is used to assess the economic consequences of increasing mandatory requirements regarding the disclosure of soft information and to investigate the firm's decision to voluntarily disclose information. ${ }^{6}$

Our results indicate that the disclosure value depends on the type of information that is to be disclosed (i.e., whether it is contractible or non-contractible information) and the amount and type of information already available to the investors. For example, the principal is, in general, indifferent regarding the disclosure of contractible information if investors have only contractible information plus no more than a single non-contractible signal. However, the principal can have strict preferences regarding the disclosure of contractible information if investors receive multiple non-contractible signals. Likewise, the principal weakly benefits from a publicly reported non-contractible performance measure if this signal is the only noncontractible information released to the investors. However, given that the investors receive non-contractible information (e.g., from analysts), the principal can be strictly worse off if she discloses a supplemental noncontractible signal.

Key to a potentially negative disclosure value is that investors' aggregation of information for pricing purposes differs from the aggregation that is optimal for the incentive contract with the agent (Paul 1992, Feltham and Xie 1994) and that the non-contractibility of information prevents the principal from adjusting and undoing investor aggregation. The difference in aggregation yields two reasons for a detrimental effect of disclosure on the principal's expected net payoff. First, while the impact of more disclosure on the sensitivity of price to the agent's action is ambiguous, disclosing more information weakly increases price volatility. Intuitively, the principal is worse off if disclosure yields a smaller signal-to-noise ratio. Secondly, disclosure can reduce the congruity of market price relative to the firm's terminal value, yielding a less

\footnotetext{
${ }^{5}$ Core, Guay and Verrecchia (2003) and Leone, Wu and Zimmerman (2006) provide empirical evidence suggesting that managerial contracts include both accounting information (e.g., annual earnings) and market price as performance measures.

${ }^{6}$ In this regard, we neglect differences between mandatory and voluntary disclosure regimes. We thank Stefan Reichelstein for pointing out this issue.
} 
efficient effort allocation across tasks. In particular, non-congruity increases if, e.g., relative to the firm's terminal value, the supplemental information is less congruent than the initial information. Importantly, disclosing contractible information affects the weights assigned by investors to the available information (including the weights assigned to the non-contractible information) ${ }^{7}$ and may result in a smaller signal-tonoise ratio or a less congruent market price. Due to the non-contractibility of some of the information that is available to the investors, the principal is unable to undo any changes to the investors' aggregation of information into price that are induced by the disclosed information. Thus, the divergence in the preferences regarding the aggregation of the performance measures combined with the non-contractibility of disclosed signals causes the negative disclosure value.

Several implications follow from our findings. First, our results are in stark contrast to the commonly held view that the usefulness of market price as a performance measure increases with the degree to which it measures firm value. For example, according to Healy and Palepu $(2001,422)$ "stock compensation is more likely to be an efficient form of remuneration for managers and owners if stock prices are a precise estimate of firm values." Secondly, our results emphasize that the value of publicly reported soft information crucially depends on the magnitude of soft information already available to investors. Then, depending on the type of incentive problem faced by firm owners, changes in mandatory disclosure requirements for soft and hard information can leave some firms worse off. Finally, non-verifiable soft information is presumably more sensitive to unproductive actions as compared with hard (accounting) information. Then, disclosing (more) soft information reduces the congruency of market price, relative to firm value. While investors in their pricing can factor out the impact of window dressing activities on their expectations of future firm value, managers will nevertheless be motivated to exert window dressing effort (Fischer and Verrecchia 2000). Assuming that earnings management is a proxy for the management of soft information, we expect to observe a positive relation between the disclosure of soft information and the extent of earnings management.

Regarding prior work on disclosure and incentive contracting, our work is most closely related to that

\footnotetext{
${ }^{7}$ Chen et al. (2006) observe that investors' reliance on other sources of information varies with corporate disclosure.
} 
of Feltham and Xie (1994), Bushman and Indjejikian (1993), and Christensen and Feltham (2000). While Feltham and Xie (1994) establish a non-negative value of additional performance measures in a multi-task setting, we find that disclosing additional contractible and non-contractible performance measures can have a negative value. Bushman and Indjejikian (1993) assume that market price includes private information to investors, and that increased disclosure can reduce investors' incentives to privately acquire information. Hence, similar to our result, increased disclosure can have a negative value in their setting. Different to our focus on the disclosure of ex post-information, Christensen and Feltham (2000) consider incentives to motivate the agent to disclose pre-decision information. Finally, our result that accounting information and stock price are only used simultaneously if stock price contains non-contractible information about the agent's action is consistent with Baiman and Verrecchia (1995).

Our work is also related to the literature on reasons for partial disclosure. In addition to explanations based on, e.g., the proprietary nature of superior information (Verrecchia 1983), uncertainty about the existence of information (Dye 1985), and uncertainty about the type of information (Teoh and Kwan 1991), our results indicate that the concurrent usage of information to address managerial incentive problems can provide a complementary rationale for partial disclosure. While the literature on partial disclosure usually discusses the ex post decision to disclose information (i.e., after the information is generated), Guay and Verrecchia (2007) show that it is valuable for the firm to commit ex ante to a full disclosure strategy if the goal is a high stock price. To the contrary, our results indicate that it can be optimal to commit ex ante to a partial disclosure strategy if the objective is to provide efficient incentives to a manager; it is straightforward that this increase in efficiency will also lead to a higher expected stock price in equilibrium.

The remainder of the paper is organized as follows. In Section 2, we present the basic model, discuss investors' pricing, and derive optimal contracts when (i) the principal uses market price as the sole performance measure, (ii) the principal uses all contractible performance measures except market price in the contract with the agent, and (iii) the principal uses all contractible performance measures plus market price in the contract with the agent. In Section 3, we determine the value of publicly reported performance measures. In Subsection 3.1, the principal discloses a single performance measure (e.g., manager's 
reputation). Subsections 3.2 and 3.3 consider the disclosure of supplemental non-contractible information (e.g., the success of adopting a new software) in a single- and multi-task setting. Finally, Subsection 3.4 analyzes disclosure value of contractible information, given that the investors also receive non-contractible information. Conclusions are presented in Section 4.

\section{BASIC NOTATION AND MODEL STRUCTURE}

\subsection{Agent's Actions, Performance Measures, and Price Formation}

At date 0 , the principal, acting on behalf of the firm's long term risk neutral owners, hires an agent to provide effort at date 1 in return for compensation at date 2 . The agent expends costly effort in $n$ tasks that influence the payoff to the principal, and his choice of effort levels is represented by the vector $\mathbf{a} \in \mathbb{R}^{n}$. We assume a linear relation between effort and the firm's terminal payoff $f^{8}$, i.e.,

$$
x=\mathbf{b}^{\prime} \mathbf{a}+\mathcal{E}_{x},
$$

where $\mathbf{b} \in \mathbb{R}^{n}$ represents the productivity of the agent's tasks and $\varepsilon_{x} \sim \mathrm{N}\left(0, \sigma_{x}^{2}\right)$ represents random events beyond the agent's control. The agent's personal cost of effort $\kappa(\mathbf{a})$ is assumed to be

$$
\kappa(\mathbf{a})=1 / 2 \mathbf{a} \mathbf{a} .
$$

At date 2, information system $\eta$ generates performance measures and releases these signals to the principal and to investors. Performance measures are either publicly reported to investors (i.e., public performance measures) or undisclosed and only released to the principal (i.e., "internal" performance measures). In addition, the information is either contractible (i.e., hard) or non-contractible (i.e., soft). Applying both classifications, we distinguish between $m_{h}$ public performance measures of hard information (denoted by the $m_{h} \times 1$ vector $\mathbf{y}_{h}$ ), $m_{s}$ public measures of soft information (denoted by the $m_{s} \times 1$ vector $\mathbf{y}_{s}$ ) and $m_{u}$ undisclosed performance measures of hard information (denoted by the $m_{u} \times 1$ vector $\mathbf{y}_{u}$ ) ${ }^{9}$ We assume a

\footnotetext{
${ }^{8}$ Alternatively, $x$ may also represent the firm's future market price. We choose the term payoff to distinguish market price at date 2 from firm value.

${ }^{9}$ Without loss in generality, we neglect undisclosed soft information. Signals including this type of information can neither be used by the principal in the contract with the agent nor by investors when updating their beliefs about the terminal payoff.
} 
linear relation between effort and performance, i.e.,

$$
\mathbf{y}_{i}=\mathbf{M}_{i} \mathbf{a}+\boldsymbol{\varepsilon}_{i}, \quad i=h, s, u,
$$

where the $m_{i} \times n$ matrix $\mathbf{M}_{i}$ represents the sensitivities of the $m_{i}$ performance measures to the agent's effort levels in his $n$ tasks, $\boldsymbol{\varepsilon}_{i}$ is a $m_{i} \times 1$ vector of normally distributed noise terms with $\boldsymbol{\varepsilon}_{i} \sim \mathrm{N}\left(\mathbf{0}, \boldsymbol{\Sigma}_{i}\right)$ and $m_{i} \times m_{i}$ covariance matrix $\boldsymbol{\Sigma}_{i}$. Moreover, $\boldsymbol{\Sigma}_{i j} \equiv \operatorname{Cov}\left[\boldsymbol{\varepsilon}_{i}, \boldsymbol{\varepsilon}_{j}\right], i, j=h, s, u$ and $i \neq j$. The noise of each performance measure reflects uncontrollable events that affect the firm's payoff plus inaccuracy of the accounting system (i.e., measurement error). Specifically, the noise is assumed to be

$$
\boldsymbol{\varepsilon}_{i}=\boldsymbol{\varepsilon}_{x} \boldsymbol{\rho}_{i}+\boldsymbol{\varepsilon}_{\mu i}, \quad i=h, s, u,
$$

where the $m_{i} \times 1$ vector $\boldsymbol{\rho}_{i}$ characterizes the correlation of the performance measures to the output, and the $m_{i} \times 1$ vector $\boldsymbol{\varepsilon}_{\mu i}$ represents measurement error, with $\boldsymbol{\varepsilon}_{\mu i} \sim \mathrm{N}\left(\mathbf{0}, \boldsymbol{\Sigma}_{\mu i}\right), \boldsymbol{\Sigma}_{\mu i}$ is the $m_{i} \times m_{i}$ covariance matrix of the measurement-related noise terms, and $\operatorname{Cov}\left[\boldsymbol{\varepsilon}_{x}, \boldsymbol{\varepsilon}_{\mu i}\right]=\mathbf{0}$. Thus $\boldsymbol{\Sigma}_{i}=\boldsymbol{\rho}_{i} \boldsymbol{\rho}_{i}{ }^{\prime} \sigma_{x}{ }^{2}+\boldsymbol{\Sigma}_{\mu i}, i=h, s, u$, where $\boldsymbol{\rho}_{i} \boldsymbol{\rho}_{i}{ }^{\prime} \sigma_{x}{ }^{2}$ is the $m_{i} \times m_{i}$ covariance matrix of the output-related noise inherent in the performance measures.

The investors can only use public information to infer the firm's terminal value. ${ }^{10}$ Likewise, the principal can only use contractible information in the agent's contract. More specifically, the investors use $\mathbf{y}_{p}=$ $\left(\mathbf{y}_{h}{ }^{\prime}, \mathbf{y}_{s}{ }^{\prime}\right)^{\prime}$ for price setting, and the principal may use $\mathbf{y}_{c}=\left(\mathbf{y}_{h}{ }^{\prime}, \mathbf{y}_{u}{ }^{\prime}\right)^{\prime}$ in the agent's contract. Then,

$$
\mathbf{y}_{l}=\mathbf{M}_{l} \mathbf{a}+\boldsymbol{\varepsilon}_{l}, \quad l=p, c,
$$

where the $m_{p} \times n$ matrix $\mathbf{M}_{p}$ represents the sensitivities of the $m_{p}=m_{h}+m_{s}$ public performance measures to the agent's effort levels, the $m_{c} \times n$ matrix $\mathbf{M}_{c}$ represents the sensitivities of the $m_{c}=m_{h}+m_{u}$ contractible performance measures to the agent's effort levels, and $\varepsilon_{l}$ is an $m_{l} \times 1$ vector of normally distributed noise terms with $\boldsymbol{\varepsilon}_{l} \sim \mathrm{N}\left(\mathbf{0}, \boldsymbol{\Sigma}_{l}\right)$ and $m_{l} \times m_{l}$ covariance matrix $\boldsymbol{\Sigma}_{l}$. The sensitivity and covariance matrices are given by the respective matrices for public and private performance measures on hard and soft information. In particular, $\boldsymbol{\rho}_{p}=\left(\boldsymbol{\rho}_{h}{ }^{\prime}, \boldsymbol{\rho}_{s}{ }^{\prime}\right)^{\prime}$ characterizes the correlation of the publicly reported performance measures with the firm's terminal payoff. ${ }^{11}$

\footnotetext{
${ }^{10}$ The signals that are made public are disclosed without additional error. This assumption is common in the literature on discretionary disclosure, where disclosure is often assumed to be truthful. For a review of this literature see Verrecchia (2001).

${ }^{11}$ Similarly, we can write $\boldsymbol{\Sigma}_{p}=\boldsymbol{\rho}_{p} \boldsymbol{\rho}_{p}{ }^{\prime} \sigma_{x}{ }^{2}+\boldsymbol{\Sigma}_{\mu p}$.
} 
The capital market is assumed to consist of homogeneous rational investors who set the price equal to the expected value of the firm conditional on all available information $\mathbf{y}_{p} \cdot{ }^{12,13}$ As the performance measures follow a normal distribution, in computing the price the investors correct the information for the unconditional mean and weight the information according to their covariance with the terminal value, scaled by the information's precision. Hence, the (gross) market price is characterized by

$$
\begin{aligned}
& \qquad \begin{array}{l}
\pi(\eta)=\mathrm{E}\left[x \mid \mathbf{y}_{p}\right]=\pi_{o}+\boldsymbol{\alpha}^{* \prime} \mathbf{y}_{p}, \\
\sigma_{\pi}^{2}(\eta) \equiv \operatorname{Var}\left[\pi \mid \mathbf{y}_{p}\right]=\operatorname{Var}\left[\boldsymbol{\alpha}^{* \prime} \mathbf{y}_{p}\right]=\sigma_{x}^{4} \boldsymbol{\rho}_{p}{ }^{\prime} \boldsymbol{\Sigma}_{p}{ }^{-1} \boldsymbol{\rho}_{p}=\sigma_{x}{ }^{2} \boldsymbol{\alpha}^{* \prime} \boldsymbol{\rho}_{p}
\end{array} \\
& \text { with } \quad \pi_{o}=\left(\mathbf{b}^{\prime}-\boldsymbol{\alpha}^{* \prime} \mathbf{M}_{p}\right) \hat{\mathbf{a}} \quad \text { and } \\
& \boldsymbol{\alpha}^{*}(\eta)=\sigma_{x}^{2} \boldsymbol{\Sigma}_{p}{ }^{-1} \boldsymbol{\rho}_{p},
\end{aligned}
$$

where $\hat{\mathbf{a}}$ denotes the investors' expectations regarding the agent's actions and the $m_{p} \times 1$ vector $\boldsymbol{\alpha}^{*}(\eta)$ represents the weights assigned to public information in the investors' price setting. Observe that the weights $\boldsymbol{\alpha}^{*}(\eta)$, market price $\pi(\eta)$, and price volatility $\sigma_{\pi}{ }^{2}(\eta)$ all vary with information system $\eta \cdot{ }^{14}$ In particular, the investors' aggregation $\boldsymbol{\alpha}^{*}(\eta)$ reflects the signals' precision (i.e., $\boldsymbol{\Sigma}_{p}^{-1}$ ) and their relation with the firm's terminal payoff(i.e., $\boldsymbol{\rho}_{p}$ ). Notably, when aggregating public signals the investors do not consider the signals' informativeness regarding the agent's actions.

\subsection{Agent's Compensation, Preferences, and Optimal Actions}

At date 0 , the principal offers the agent a linear contract $z=\left(f, v_{\pi}, \mathbf{v}_{c}\right)$, where $f$ is the agent's fixed wage, $v_{\pi}$ the incentive rate for market price, and $\mathbf{v}_{c}$ an $m_{c} \times 1$ vector of incentive rates for any additional contractible signal. Hence, the agent's compensation $w$, given price $\pi$, contractible information $\mathbf{y}_{c}$, and contract $z$ is

$$
w\left(\pi, \mathbf{y}_{c}, z\right)=f+v_{\pi} \pi+\mathbf{v}_{c} \mathbf{y}_{c} .
$$

${ }^{12}$ This setting is equivalent to Feltham and Xie (1994) and similar to the market maker's behavior in Kyle (1985). Note that our results hold under the assumption of an incomplete market with private information amongst investors. Then, the information that is impounded into price through the trading decisions of informed investors can be viewed as public soft information.

${ }^{13}$ A possible question at this point refers to the consequences if the principal (strategically) chooses not to release some signals (we thank Stan Baiman for this observation). The capital market participants know about the set of public signals. In our setting, the principal decides about the disclosure before she learns the outcome of the performance measures. Thus, by observing the disclosure decision, investors learn nothing regarding the realization of the signals.

${ }^{14}$ However, in order to simplify notation, whenever the result is unambiguous we omit the reference regarding the impact of information system $\eta$ on $\boldsymbol{\alpha}^{*}, \pi$, and $\sigma_{\pi}^{2}$. 
We assume that the agent's preferences are represented by a negative exponential utility function, with

$$
u(z, \mathbf{a})=-\exp [-r(w-\kappa(\mathbf{a}))]
$$

where $r$ is the coefficient of his absolute risk aversion.

Maximizing the expected utility is equivalent to maximizing the certainty equivalent, which is characterized by

$$
\begin{aligned}
\mathrm{CE}(z, \mathbf{a}) & =\mathrm{E}[w \mid z, \mathbf{a}]-\kappa(\mathbf{a})-1 / 2 r \operatorname{Var}[w \mid z] \\
& =f+v_{\pi}\left(\left(\mathbf{b}^{\prime}-\boldsymbol{\alpha}^{* \prime} \mathbf{M}_{p}\right) \hat{\mathbf{a}}+\boldsymbol{\alpha}^{* \prime} \mathbf{M}_{p} \mathbf{a}\right)+\mathbf{v}_{c}{ }^{\prime} \mathbf{M}_{c} \mathbf{a}-1 / 2 \mathbf{a} \mathbf{a}^{\prime} \mathbf{a}-1 / 2 r\left(v_{\pi}{ }^{2} \sigma_{\pi}{ }^{2}+\mathbf{v}_{c}{ }^{\prime} \mathbf{\Sigma}_{c} \mathbf{v}_{c}+2 v_{\pi} \boldsymbol{\sigma}_{\pi c}{ }^{\prime} \mathbf{v}_{c}\right)
\end{aligned}
$$

where $\boldsymbol{\sigma}_{\pi c} \equiv \operatorname{Cov}\left[\pi, \mathbf{y}_{c}\right]$ is an $m_{c} \times 1$ vector of correlations between price $\pi$ and contractible performance measures $\mathbf{y}_{c}$.

The agent chooses a to maximize his certainty equivalent. Differentiating (2) with respect to a, given contract $z$, provides the following characterization of the agent's action choices.

Lemma 1: Given contract $z=\left(f, v_{\pi}, \mathbf{v}_{c}\right)$, the agent's optimal action choices are characterized by

$$
\mathbf{a}^{\dagger}\left(v_{\pi}, \mathbf{v}_{c}\right)=\mathbf{M}_{p}{ }^{\prime} \boldsymbol{\alpha}^{*} v_{\pi}+\mathbf{M}_{c}{ }^{\prime} \mathbf{v}_{c}
$$

Proof: Differentiating (2) with respect to a, and solving the first-order conditions for this variable results in the optimal action choices as specified in (3).

Observe that by selecting $\mathbf{v}_{c}$, the principal can vary the relative weight for any contractible information. On the other hand, the weights for non-contractible information (i.e., the weights for soft information that is included in $\mathbf{y}_{p}$ ) are given by the investors' aggregation (i.e., $\boldsymbol{\alpha}^{*}$ ). With respect to these signals, by selecting $v_{\pi}$ the principal can only vary the intensity of the incentives.

\subsection{Principal's Contract Choice}

When the principal offers contract $z$ to the agent, she has to take into account that the agent provides unobservable effort and chooses whether to accept the contract. The actions that are induced by $z$ are characterized by (3). Also, the agent will only participate in the firm if his contract $z$ is such that it provides him with his reservation wage, which is scaled to equal zero, i.e.,

$$
C E\left(z, \mathbf{a}^{\dagger}\right) \geq 0
$$


The principal is assumed to be risk neutral with respect to the terminal value of the firm. She is interested in maximizing the expected terminal value of the firm net of the agent's compensation ${ }^{15}$, i.e.,

$$
\Pi(\mathbf{a}, z, \eta)=\mathrm{E}[x-w \mid \mathbf{a}, z, \eta]
$$

The principal chooses $z$ such as to maximize (5) subject to (4) and (3). Substituting (3) into (2), choosing $f$ such that $C E\left(z, \mathbf{a}^{\dagger}\right)=0$, and substituting $f^{\dagger}$ and (3) into (5) gives the principal's unconstrained decision problem:

$$
\begin{aligned}
\Pi\left(v_{\pi}, \mathbf{v}_{c}, \eta\right)= & \mathbf{b}^{\prime}\left(\mathbf{M}_{p}{ }^{\prime} \boldsymbol{\alpha}^{*} v_{\pi}+\mathbf{M}_{c}{ }^{\prime} \mathbf{v}_{c}\right)-1 / 2\left(\mathbf{M}_{p}{ }^{\prime} \boldsymbol{\alpha}^{*} v_{\pi}+\mathbf{M}_{c}{ }^{\prime} \mathbf{v}_{c}\right)^{\prime}\left(\mathbf{M}_{p}{ }^{\prime} \boldsymbol{\alpha}^{*} v_{\pi}+\mathbf{M}_{c}{ }^{\prime} \mathbf{v}_{c}\right) \\
& -1 / 2 r\left(v_{\pi}{ }^{2} \sigma_{\pi}{ }^{2}+\mathbf{v}_{c}{ }^{\prime} \mathbf{\Sigma}_{c} \mathbf{v}_{c}+2 v_{\pi} \boldsymbol{\sigma}_{\pi c}{ }^{\prime} \mathbf{v}_{c}\right)
\end{aligned}
$$

To characterize the solution to the principal's decision problem, we show the results for the setting where (i) the principal uses market price $\pi$ as the sole performance measure, (ii) she only contracts on the contractible performance measures $\mathbf{y}_{c}$, and (iii) she uses market price and the contractible performance measures in the agent's contract. Proposition 1 summarizes the results. ${ }^{16}$

\section{Proposition 1:}

(i) Suppose that the principal uses market price as the sole performance measure (i.e., the contract just includes $\pi$ ); the optimal incentive rate and the principal's expected net payoff are characterized by

$$
\begin{aligned}
& v_{\pi p}{ }^{\dagger}=Q_{p}{ }^{-1} \mathbf{b}^{\prime} \mathbf{M}_{p}{ }^{\prime} \boldsymbol{\alpha}^{*}, \\
& \Pi_{p}^{\dagger}(\eta)=1 / 2 Q_{p}{ }^{-1}\left(\mathbf{b}^{\prime} \mathbf{M}_{p}{ }^{\prime} \boldsymbol{\alpha}^{*}\right)^{2},
\end{aligned}
$$

with $Q_{p}=\boldsymbol{\alpha}^{* \prime} \mathbf{M}_{p} \mathbf{M}_{p}{ }^{\prime} \boldsymbol{\alpha}^{*}+r \sigma_{\pi}^{2}$.

(ii) Suppose that the principal uses all contractible performance measures except market price in the agent's contract (i.e., the contract includes $\mathbf{y}_{c}$ ); the optimal incentive rates and the principal's expected net payoff are characterized by

\footnotetext{
${ }^{15} \mathrm{We}$ assume the principal to act in the interest of the long-term shareholders. The latter are not interested in short-term prices but in the firm's terminal value. The same assumption is made by Baiman and Verrecchia (1995) and Feltham and Xie (1994).

${ }^{16}$ The propositions are proven in Appendix A. While setting (ii) is identical to Section I in Feltham and Xie (1994), setting (i) is identical to their Section V.
} 


$$
\begin{aligned}
& \mathbf{v}_{c y}^{\dagger}=\mathbf{Q}^{-1} \mathbf{M}_{c} \mathbf{b}, \\
& \Pi_{y}^{\dagger}(\eta)=1 / 2 \mathbf{b}^{\prime} \mathbf{M}_{c}{ }^{\prime} \mathbf{Q}^{-1} \mathbf{M}_{c} \mathbf{b},
\end{aligned}
$$

with $\mathbf{Q} \equiv \mathbf{M}_{c} \mathbf{M}_{c}{ }^{\prime}+r \boldsymbol{\Sigma}_{c}$.

(iii) Suppose that the principal uses all contractible performance measures including market price in the agent's contract (i.e., the contract includes $\mathbf{y}_{c}$ and $\pi$ ); the optimal incentive rates and the principal's expected net payoff are characterized by

$$
\begin{aligned}
& {\left[\begin{array}{c}
v_{\pi}^{\dagger} \\
\mathbf{v}_{c}^{\dagger}
\end{array}\right]=\left[\begin{array}{cc}
\boldsymbol{\alpha}^{* \prime} \mathbf{M}_{p} \mathbf{M}_{p}{ }^{\prime} \boldsymbol{\alpha}^{*}+r \sigma_{\pi}^{2} & \boldsymbol{\alpha}^{* \prime} \mathbf{M}_{p} \mathbf{M}_{c}{ }^{\prime}+r \boldsymbol{\sigma}_{\pi c}{ }^{\prime} \\
\mathbf{M}_{c} \mathbf{M}_{p}{ }^{\prime} \boldsymbol{\alpha}^{*}+r \boldsymbol{\sigma}_{\pi c} & \mathbf{M}_{c} \mathbf{M}_{c}+r \boldsymbol{\Sigma}_{c}
\end{array}\right]^{-1}\left[\begin{array}{c}
\mathbf{b}^{\prime} \mathbf{M}_{p}{ }^{\prime} \boldsymbol{\alpha}^{*} \\
\mathbf{M}_{c} \mathbf{b}
\end{array}\right] \equiv \mathbf{G}^{-1} \underline{\mathbf{b}},} \\
& \Pi^{\dagger}(\eta)=1 / 2 \underline{\mathbf{b}} \mathbf{G}^{-1} \underline{\mathbf{b}} .
\end{aligned}
$$

In setting (i), while there are multiple public performance measures, the principal just uses market price $\pi$. The relative weights of the public signals in the price formation are given by the aggregation $\boldsymbol{\alpha}^{*}$ chosen by investors when inferring the firm's terminal value. Note that the distinction between soft and hard information does not influence investors' inference regarding the firm's terminal value. Moreover, using market price, the principal can indirectly contract on non-contractible information. Also, from (7a) and (7b), the aggregation $\boldsymbol{\alpha}^{*}$ is key to the agent's incentive rate and the principal's expected net payoff. In particular, if the publicly reported signals are not related to the firm's terminal value (i.e., if $\boldsymbol{\rho}_{p}=\mathbf{0}$, implying $\boldsymbol{\alpha}^{*}=\mathbf{0}$ ), the principal refrains from motivating the agent's actions by means of incentive compensation.

In setting (ii), the principal does not use market price. Results (7c) and (7d) reveal that the investors' aggregation of public information does not affect the incentive rates offered to the agent and the principal's expected net payoff. Rather, the principal has discretion with respect to selecting the relative weights for contractible information in the agent's contract. Following Feltham and Xie (1994), the relative incentive rates reflect the performance measures' precision and their congruency with the firm's terminal value. In particular, the weights are not affected by the correlation between the performance measures and the firm's terminal value.

Finally, setting (iii) is a hybrid between (i) and (ii) in that the principal uses all contractible performance 
measures including market price in the agent's contract. Following (7e), in general, the investors' aggregation $\boldsymbol{\alpha}^{*}$ affects all incentive rates, i.e., it also affects the (relative) weights assigned to contractible information. Therefore, the characteristics of non-contractible information will influence the incentive rates chosen for contractible performance measures. Similar to setting (i), the principal's expected net payoff will depend on pricing (i.e., $\boldsymbol{\alpha}^{*}$ ); in particular, it will reflect characteristics of disclosed soft information.

To summarize, given that the principal uses market price when contracting with the agent (i.e., settings (i) and (iii)), the aggregation of public signals by investors directly affects the principal's expected net payoff. Importantly, when aggregating public information, the investors are ignorant regarding the impact of their aggregation on the principal's surplus.

\section{VALUE OF DISCLOSING PERFORMANCE MEASURES TO INVESTORS}

Notably, for the three settings of Proposition 1, the investors' aggregation of public signals (i.e., $\boldsymbol{\alpha}^{*}$ ) is unaffected by the performance measures that are selected by the principal. On the other hand, varying the set of performance measures that are reported to investors will, in general, alter aggregation $\boldsymbol{\alpha}^{*}$ and, in settings (i) and (iii), affect the optimal incentive rates plus the principal's expected net payoff. Given the investors' ignorance regarding the impact of their pricing (i.e., aggregation of public information) on the principal's expected net payoff, the value of disclosing information to investors is an open question.

Let $\eta_{o}$ represent the initial information system with a set of "internally" released performance measures (i.e., $\left.\mathbf{Y}_{i}=\left\{y_{i 1}, \ldots, y_{i m_{i}}\right\}\right)$ and a set of publicly reported performance measures (i.e., $\mathbf{Y}_{p}=\left\{y_{p 1}, \ldots, y_{p m_{p}}\right\}$ ). Next, consider $\eta_{s}$ that is identical to $\eta_{o}$ except for an additional, previously internal and now publicly reported performance measure $y_{s} \in \mathbf{Y}_{i}$. With $\eta_{o}$, if $y_{s}$ is hard information, the signal is (most likely) included in the agent's contract; if it is soft information, we assume that the signal is generated even though it is not used by the principal. Therefore, $\eta_{s}$ is characterized by $\mathbf{Y}_{i}{ }^{\prime}$ and $\mathbf{Y}_{p}{ }^{\prime}$, with $\mathbf{Y}_{i}{ }^{\prime}=\mathbf{Y}_{i} \backslash y_{s}$ and $\mathbf{Y}_{p}{ }^{\prime}=\mathbf{Y}_{p} \cup y_{s}$.

The value of disclosing $y_{s}$ to investors (i.e., the disclosure value) follows from comparing the expected net payoff to the principal under $\eta_{o}$ and $\eta_{s}$. In particular: 
Definition 1: The value of an additional, publicly reported performance measure $y_{s}$ is given by

$$
V^{\dagger}\left(y_{s} \mid \eta_{o}\right) \equiv \Pi^{\dagger}\left(\eta_{s}\right)-\Pi^{\dagger}\left(\eta_{o}\right)
$$

Following (8), in general, the disclosure value of the supplemental performance measure will depend on the nature of the incentive problem, the characteristics of $y_{s}$, and the set of further disclosed performance measures (characterized by $\eta_{o}$ ). More specifically, the value will depend, among other variables, on the agent's risk aversion, the assignment of a single or multiple tasks to the agent, the contractibility of supplemental information (i.e., whether $y_{s}$ is hard or soft information), the number and characteristics of further public performance measures, i.e., the contractibility of additionally disclosed information.

To gain insight into the value of an additional, publicly reported performance measure, we subsequently consider several special cases. In Section 3.1, we consider the baseline setting where the performance measure under study is the only information that is released to investors. While Section 3.2 considers the disclosure of supplemental non-contractible information in a single-task setting, Section 3.3 addresses the value of supplemental non-contractible information in a multi-task setting. Finally, Section 3.4 considers the disclosure value of contractible information, given that non-contractible information is publicly reported. In the latter three sections, we first identify conditions under which the supplemental signal is price relevant and then highlight conditions where disclosure leads to a loss to the principal.

\subsection{Disclosure of a Single Performance Measure}

To illustrate the value of disclosing a single type of information to investors, the simplest case to consider is one in which the agent undertakes a single task and there is one performance measure. More specifically, $y_{1}=m a+\rho_{1 x} \mathcal{E}_{x}+\mathcal{E}_{\mu 1}$, where $\rho_{1 x} \mathcal{E}_{x}$ is output-related fluctuation reflected in $y_{1}$ and $\mathcal{E}_{\mu 1}$ is measurement noise, with $\varepsilon_{x} \sim \mathrm{N}\left(0, \sigma_{x}^{2}\right), \mathcal{\varepsilon}_{\mu 1} \sim \mathrm{N}\left(0, \sigma_{\mu 1}{ }^{2}\right)$, and $\operatorname{Cov}\left[\mathcal{E}_{\mu 1}, \mathcal{\varepsilon}_{x}\right]=0 ; \sigma_{1}{ }^{2} \equiv \operatorname{Var}\left[y_{1}\right]=\rho_{1 x}{ }^{2}{\sigma_{x}}^{2}+{\sigma_{\mu 1}}^{2}$ is the variance of performance measure $y_{1}$. Under $\eta_{0}$, no information regarding the firm's terminal payoff is publicly reported. On the other hand, under $\eta_{1}, y_{1}$ is disclosed to investors. While the agent's compensation is $w=f+v_{1} y_{1}$ under $\eta_{0}$, it is $w=f+v_{1} y_{1}+v_{\pi} \pi$ under $\eta_{1}$.

Table 1 uses this parameterization in (7a) to (7d) to characterize the incentive rates and the expected net payoff to the principal, assuming that $y_{1}$ is either hard (Panel A) or soft information (Panel B). 
Given that $y_{1}$ is hard information, Panel A of Table 1 illustrates that, following (T1.2b), while disclosing $y_{1}$ affects investors' inference of the firm's terminal value and, thus, market price (i.e., $\pi\left(\hat{a}, y_{1}\right)=\left(b-\alpha^{*} m\right) \hat{a}$ $+\alpha^{*} y_{1}$ ), the disclosure does not influence the principal's solution to the incentive problem (compare (T1.1c) with (T1.2c)). Key to this result is that $\pi\left(y_{1}\right)$ is an equivalent statistic to $y_{1}$, i.e., $\pi$ is invertible into $y_{1}$. Consequently, Panel A describes one solution out of an infinite number of solutions that are equivalent in terms of the principal's expected net payoff; namely, the principal does not use price in the agent's contract. ${ }^{17}$

On the other hand, Panel B illustrates that the principal cannot directly use the performance measure if $y_{1}$ is soft information. Hence, if, under $\eta_{0}, y_{1}$ is not disclosed, the principal cannot motivate the agent to exert effort such that her expected net payoff as well as the market price are equal to zero. To the contrary, with $\eta_{1}$, i.e., given that non-contractible information is disclosed, $y_{1}$ is impounded in price. Hence, the principal can indirectly contract on $y_{1}$. In particular, following (T1.4b), $y_{1}$ is impounded in price if it is assigned a nonzero weight $\alpha^{*}$, i.e., if it is informative about uncontrollable events that affect the firm's terminal payoff (i.e., if $\rho_{1 x} \neq 0$ ). Following (T1.4c) and (T1.4d), disclosing $y_{1}$ enables the principal to induce agent effort and results in a positive expected net payoff to the principal. ${ }^{18}$

Proposition 2 summarizes the results regarding the disclosure value of a single performance measure.

Proposition 2: Disclosing a single performance measure is weakly beneficial to the principal. In particular:

(i) Disclosing hard information has no value to the principal (i.e., $V^{\dagger}\left(y_{1} \mid \eta_{0}\right)=0$ );

(ii) Disclosing soft information is strictly valuable to the principal if it is informative about uncontrollable events that affect the firm's terminal payoff (i.e., $V^{\dagger}\left(y_{1} \mid \eta_{0}\right)>0$ if $\rho_{1 x} \neq 0$ ).

The proof follows directly from substituting (T1.1d) and (T1.2d), or (T1.3d) and (T1.4d) into (8).

Following Proposition 2, disclosing a single performance measure is not harmful to the principal. More

\footnotetext{
${ }^{17}$ The infinite number of solutions are characterized by $\left(v_{1}^{\dagger}, v_{\pi}^{\dagger}\right)=\left\{\left(v_{1}, v_{\pi}\right) \in \mathbb{R}^{2} \mid v_{1}+\alpha^{*} v_{\pi}=\left(m^{2}+r \sigma_{1}{ }^{2}\right)^{-1} b m\right\}$.

${ }^{18}$ Interestingly, since price is an equivalent statistic to soft information, the expected net payoff to the principal is identical to the expected surplus in the setting with hard information (compare (T1.2d) with (T1.4d)).
} 
specifically, while the principal is indifferent with respect to disclosure if $y_{1}$ is hard information, she has strict preferences for disclosure if $y_{1}$ is soft information. Key to this result is that under $\eta_{0}$ market price is not used in the agent's contract. Therefore, while disclosing $y_{1}$ affects investors' inference regarding the firm's terminal payoff and, thus, market price, by setting $v_{\pi}=0$ under $\eta_{1}$ the principal can always mimic the solution to the incentive problem under $\eta_{0}$. Hence, there is no downside in disclosing $y_{1}$.

Notably, this result generalizes to settings where $\eta_{0}$ generates multiple performance measures but discloses no information and where $\eta_{1}$ is identical to $\eta_{0}$ except that it publicly reports either one or several of the performance measures that are generated by $\eta_{0}$. Likewise, the result also generalizes to settings where multiple tasks are assigned to the agent and contractible information is publicly reported. Intuitively, both, the disclosure of a single or multiple performance measures result in a market price that varies with disclosed information, thus introducing an option for the principal to indirectly contract on this information; on the other hand, abandoning the option does not prevent the principal from offering the same incentive contract to the agent as under $\eta_{0}$.

\subsection{Disclosing Supplemental Non-contractible Information in a Single-task Setting}

The preceding analysis provides a simple model to illustrate that disclosing a single performance measure is weakly beneficial to the principal. In this subsection we consider a simple setting where disclosing supplemental, non-contractible information can be detrimental to the principal. More specifically, we identify conditions under which additional, publicly reported soft information is detrimental to the principal.

To illustrate this result, we extend the previous model by assuming that there are two non-contractible performance measures $y_{i}=m a+\rho_{i x} \mathcal{E}_{x}+\mathcal{E}_{\mu i}, i=1,2$, where $\mathcal{E}_{\mu i} \sim \mathrm{N}\left(0, \sigma_{\mu i}{ }^{2}\right), \operatorname{Cov}\left[\mathcal{E}_{\mu i}, \mathcal{E}_{x}\right]=0$, and $\operatorname{Cov}\left[\mathcal{E}_{\mu 1}, \mathcal{E}_{\mu 2}\right]=$ $\rho \sigma_{\mu 1} \sigma_{\mu 2}{ }^{19}$ While $\eta_{1}$ only discloses the initial performance measure (i.e., $y_{1}$ ), $\eta_{2}$ also discloses a supplemental performance measure (i.e., $y_{1}$ and $\left.y_{2}\right)$. Thus, $V^{\dagger}\left(y_{2} \mid \eta_{1}\right)$ reflects the disclosure value of supplemental noncontractible information (i.e., the value of additional, publicly reported soft information). Suppose that the

\footnotetext{
${ }^{19}$ Assuming that $y_{2}$ has the same sensitivity to the agent's action as $y_{1}$ (i.e., $\partial \mathrm{E}\left[y_{1}\right] / \partial a=\partial \mathrm{E}\left[y_{2}\right] / \partial a$ ) is without loss in generality, since we can always generate an equivalent statistic $\psi_{2}$ to $y_{2}{ }^{\prime}=m^{\prime} a+\rho_{2 x} \mathcal{E}_{x}+\mathcal{E}_{2}$ with an arbitrary $m^{\prime} \in \mathbb{R}$, such that $\psi_{2}$ has the same sensitivity as $y_{1}$.
} 
supplemental signal is (conditionally) informative about the agent's actions. Moreover, if this signal is also contractible, using it in the contract with the agent is (weakly) beneficial to the principal. However, additional, publicly reported non-contractible information can yield starkly different results.

\subsubsection{Price-relevance}

Even if the supplemental signal is (conditionally) informative about the agent's action, investors will not always use this information in their pricing. Lemma 2 summarizes conditions under which $y_{2}$ is not used in the price setting process.

Lemma 2: Investors do not use supplemental information $y_{2}$ to set the price if $\left(\rho_{2 x} \sigma_{\mu 1}-\rho \rho_{1 x} \sigma_{\mu 2}\right) \sigma_{\mu 1}=$

0. This condition holds, for example, if:

(i) both signals are not informative about the firm's terminal payoff (i.e., $\rho_{1 x}=\rho_{2 x}=0$ );

(ii) the supplemental signal is not informative about either the firm's terminal payoff or the measurement error of the initial signal (i.e., $\rho_{2 x}=\rho=0$ );

(iii) the initial signal is informative about the firm's terminal payoff and contains no measurement error (i.e., $\rho_{1 x} \neq 0$ and ${\sigma_{\mu 1}}^{2}=0$ );

(iv) both signals are equally informative about the firm's terminal payoff and the conditional measurement error of the initial signal is zero (i.e., $\rho_{1 x}=\rho_{2 x}$ and $\sigma_{\mu 1}-\rho \sigma_{\mu 2}=0$ ).

In general, $y_{2}$ is not price relevant if $y_{1}$ is a sufficient statistic for $y_{1}$ and $y_{2}$ with respect to $x$. In particular, in (i) and (ii), $y_{2}$ is not informative about the firm's terminal payoff. Then, from the market's perspective, its only purpose is to reduce measurement noise that is included in $y_{1}$. However, if $y_{1}$ is not used in the first place (i.e., $\rho_{1 x}=0$ ) or $y_{2}$ is not informative about $y_{1}$ 's measurement error $(\rho=0), y_{2}$ will not be considered in price setting. Interestingly, this includes the special case of a noiseless performance measure (i.e., $\sigma_{2}^{2}=0$ ). Case (iii) characterizes a setting where $y_{1}$ perfectly reveals $x$ such that, consequently, price perfectly reflects terminal payoffs. Finally, case (iv) describes a setting where $y_{2}$ is merely a garbling of $y_{1}$.

If any condition outlined in Lemma 2 holds, $y_{2}$ is not used to derive the firm's price. Then, given that $y_{2}$ is non-contractible information, the principal can neither directly nor indirectly use the supplemental performance measure in the contract with the agent. 
Assuming that neither condition outlined in Lemma 2 holds, using the model's parameterization in (1a) and (1b) gives the sensitivity of market price to the agent's action (i.e., $\partial \mathrm{E}\left[\pi \mid \eta_{2}\right] / \partial a$ ) and price volatility (i.e., $\left.\sigma_{\pi}^{2}\left(\eta_{2}\right)\right)$, which are characterized by

$$
\begin{aligned}
& \partial \mathrm{E}\left[\pi \mid \eta_{2}\right] / \partial a=Q^{-1} m\left(\rho_{1 x} \sigma_{\mu 2}^{2}-\rho\left(\rho_{1 x}+\rho_{2 x}\right) \sigma_{\mu 1} \sigma_{\mu 2}+\rho_{2 x} \sigma_{\mu 1}^{2}\right) \sigma_{x}^{2}, \\
& \sigma_{\pi}^{2}\left(\eta_{2}\right)=Q^{-1}\left(\rho_{1 x}^{2} \sigma_{\mu 2}^{2}-2 \rho \rho_{1 x} \rho_{2 x} \sigma_{\mu 1} \sigma_{\mu 2}+\rho_{2 x}{ }^{2} \sigma_{\mu 1}{ }^{2}\right) \sigma_{x}^{4}
\end{aligned}
$$

where $Q \equiv\left(\rho_{1 x}{ }^{2} \sigma_{x}{ }^{2}+\sigma_{\mu 1}{ }^{2}\right)\left(\rho_{2 x}{ }^{2} \sigma_{x}^{2}+\sigma_{\mu 2}{ }^{2}\right)-\left(\rho_{1 x} \rho_{2 x} \sigma_{x}^{2}+\rho \sigma_{\mu 1} \sigma_{\mu 2}\right)^{2}$. Observe that both figures are key to the solution of the principal's decision problem under $\eta_{2}$.

\subsubsection{Disclosure Value}

Since both performance measures $y_{i}, i=1,2$, are non-contractible, the solution to the principal's problem with $\eta_{1}$ is the same as the solution in Section 3.1 (see Panel B in Table 1). In particular, the principal's expected net payoff is characterized by (T1.4d). On the other hand, using the parameterization for $\eta_{2}$ in (7b) gives the principal's expected net payoff as

$$
\Pi^{\dagger}\left(\eta_{2}\right)=1 / 2 \frac{b^{2} m^{2}}{m^{2}+r \sigma_{\pi}^{2}\left(\frac{m}{\partial \mathrm{E}\left[\pi \mid \eta_{2}\right] / \partial a}\right)^{2}}
$$

where $\partial \mathrm{E}\left[\pi \mid \eta_{2}\right] / \partial a$ is given by (9a) and $\sigma_{\pi}^{2}$ is given by (9b). Substituting (T1.4d) and (10) into (8) and simplifying yields the value of disclosing $y_{2}$, given disclosure of $y_{1}$, as

$$
V^{\dagger}\left(y_{2} \mid \eta_{1}\right)=1 / 2 \frac{b^{2} r \sigma_{1}^{2} \sigma_{\pi}^{2}}{\left(\left(\partial \mathrm{E}\left[\pi \mid \eta_{2}\right] / \partial a\right)^{2}+r \sigma_{\pi}^{2}\right)\left(m^{2}+r \sigma_{1}^{2}\right)}\left(\frac{\left(\partial \mathrm{E}\left[\pi \mid \eta_{2}\right] / \partial a\right)^{2}}{\sigma_{\pi}^{2}}-\frac{m^{2}}{\sigma_{1}^{2}}\right) .
$$

Suppose that the conditions outlined in Lemma 2 do not hold, i.e., supplemental information is impounded in price. Then, following (11), the sign of its disclosure value depends on the market prices' signal-to-noise ratios under $\eta_{2}$ versus $\eta_{1}$ (Kim and Suh 1991; Lambert 2001). In particular, disclosing supplemental information is beneficial to the principal if the signal-to-noise ratio with $\eta_{2}$ is larger than the signal-to-noise ratio with $\eta_{1}$ (i.e., if $\partial \mathrm{E}\left[\pi \mid \eta_{2}\right] / \partial a / \sigma_{\pi}>m / \sigma_{1}$ ). In general, given that the supplemental information is impounded in price, disclosure of $y_{2}$ increases price volatility (i.e., $\sigma_{\pi}^{2}\left(\eta_{2}\right)>\sigma_{\pi}^{2}\left(\eta_{1}\right)=\sigma_{1}^{2}$ if Lemma 2 does not hold), whereas the impact of disclosure on the sensitivity of market price to the agent's 
action is ambiguous. Proposition 3 summarizes necessary and sufficient conditions under which disclosing supplemental information $y_{2}$ is detrimental to the principal.

Proposition 3: A divergent informativeness of the initial versus the supplemental performance measure regarding the firm's terminal payoff is a necessary condition for a negative value of supplemental, publicly reported information (i.e., $\rho_{1 x} \neq \rho_{2 x}$ ). Sufficient conditions for a negative disclosure value include, for example, modest measurement error in the initial performance measure and a relatively more payoffinformative supplemental performance measure with

(i) no measurement error (i.e., ${\sigma_{\mu 1}}^{2}<\left(1-\rho_{1 x}{ }^{2}\right){\sigma_{x}}^{2}, \rho_{2 x}{ }^{2}>\rho_{1 x}{ }^{2}+{\sigma_{\mu 1}}^{2} / \sigma_{x}^{2}$, and $\sigma_{\mu 2}=0$ );

(ii) unrelated measurement error (i.e., ${\sigma_{\mu 1}}^{2}<\left(1-2 \rho_{1 x} / \rho_{2 x}\right) \sigma_{\mu 2}{ }^{2}+\left(\rho_{2 x}{ }^{2}-\rho_{1 x}{ }^{2}\right) \sigma_{x}{ }^{2}, \rho_{2 x}>2 \rho_{1 x}>0$, and $\rho=0$ ).

In settings (i) and (ii), volatility increases with disclosure because (a) the initial performance measure is subject to only modest measurement error and (b) the supplemental performance measure is substantially more informative about terminal payoffs than the initial signal. ${ }^{20}$ If the supplemental performance measure is substantially more informative about the firm's terminal payoff than the initial performance measure, investors assign a relatively larger weight to the former signal (i.e., $\alpha_{2}{ }^{*}$ versus $\alpha_{1}{ }^{*}$ ), yielding a lower sensitivity of price to the agent's action. Consequently, the additional, publicly reported signal decreases the signal-tonoise ratio of market price and, according to (11), has a negative disclosure value.

Fundamental to Proposition 3 is that investors are ignorant regarding the impact of their pricing on the principal's expected net payoff. Intuitively, whereas the principal would assign a relatively larger weight to $y_{1}$ (because of its modest measurement error), investors place a relatively larger weight on $y_{2}$ (because of its higher payoff informativeness). It is this divergence in the aggregation of the performance measures (Paul 1992, Feltham and Xie 1994) that causes the negative disclosure value. Interestingly, this chain of reasoning holds for the case where the supplemental signal possesses no measurement error (Proposition 3 (i)) and the case where its measurement error is unrelated to the measurement error of the initial signal (Proposition 3

\footnotetext{
${ }^{20}$ Observe that volatility increases in the performance measure's payoff informativeness. Intuitively, for a more informative signal, price varies to a larger extent with the disclosed signal.
} 
(ii)). ${ }^{21}$

Observe that Proposition 3 holds as long as market price is used as the sole performance measure in the agent's contract. Key to this result is that investors aggregate information regardless of its contractibility, i.e., the weight $\alpha_{i}^{*}$ assigned to $y_{i}$ is not affected by this signal's contractibility. On the other hand, if either the initial or the supplemental performance measure is contractible, disclosure value for the supplemental signal will be non-negative. With at least one contractible performance measure, by varying the incentive rate for this signal the principal can induce any relative weights for both, the contractible signal and market price, including the weights that she would choose if both signals were contractible.

\subsection{Disclosing Supplemental Non-contractible Information in a Two-task Setting}

We now consider a simple model that starkly illustrates how, in a two-task setting, supplemental, publicly reported non-contractible information can be detrimental to the principal. Unlike the previous model, we assume that two actions $a_{1}$ and $a_{2}$ influence two aggregate, non-contractible performance measures $y_{i}=m_{i 1} a_{1}$ $+m_{i 2} a_{2}+\rho_{i x} \mathcal{E}_{x}+\mathcal{E}_{\mu i}, \quad i=1,2$, where $\mathcal{E}_{\mu i} \sim \mathrm{N}\left(0, \sigma_{\mu i}{ }^{2}\right), \operatorname{Cov}\left[\mathcal{E}_{\mu i}, \mathcal{E}_{x}\right]=0$, and $\operatorname{Cov}\left[\mathcal{E}_{\mu 1}, \mathcal{E}_{\mu 2}\right]=\rho \sigma_{\mu 1} \sigma_{\mu 2}{ }^{22}$ As in the previous model, while $\eta_{1}$ only discloses an initial performance measure (i.e., $y_{1}$ ), $\eta_{2}$ also discloses a supplemental performance measure (i.e., $y_{1}$ and $\left.y_{2}\right)$. Hence, $V^{\dagger}\left(y_{2} \mid \eta_{1}\right)$ continues to reflect the disclosure value of supplemental non-contractible information.

Note that Lemma 2 continues to apply in this setting. Key to this result is that investors are only interested in the signals' payoff-informativeness, which is not affected by the agent's effort choices. More generally, while the weight for $y_{1}$ under $\eta_{1}$ is given by $\alpha^{*}=\left(\rho_{1 x}{ }^{2}+\sigma_{\mu 1}{ }^{2} / \sigma_{x}{ }^{2}\right)^{-1} \rho_{1 x}$ (see Table 1$)$, using (1c), with $\eta_{2}$ the weights for both signals are given by

$$
\begin{aligned}
& \alpha_{1}{ }^{*}=Q^{-1}\left(\rho_{1 x} \sigma_{\mu 2}-\rho \rho_{2 x} \sigma_{\mu 1}\right) \sigma_{\mu 2} \sigma_{x}^{2}, \\
& \alpha_{2}{ }^{*}=Q^{-1}\left(\rho_{2 x} \sigma_{\mu 1}-\rho \rho_{1 x} \sigma_{\mu 2}\right) \sigma_{\mu 1} \sigma_{x}^{2},
\end{aligned}
$$

where $Q \equiv\left(\rho_{1 x}{ }^{2} \sigma_{x}^{2}+\sigma_{\mu 1}{ }^{2}\right)\left(\rho_{2 x}{ }^{2} \sigma_{x}{ }^{2}+\sigma_{\mu 2}{ }^{2}\right)-\left(\rho_{1,} \rho_{2 x} \sigma_{x}{ }^{2}+\rho \sigma_{\mu 1} \sigma_{\mu 2}\right)^{2}$. Applying these weights to the performance

\footnotetext{
${ }^{21}$ Note that these additional conditions affect the cutoff-values for the measurement error of the initial signal and the supplemental signal's payoff informativeness.

${ }^{22}$ For simplicity, $m_{i j} \geq 0, i, j=1,2$.
} 
measures determines how the agent's actions influence market price.

Subsequently, to illustrate our results, we first consider a setting where the agent is risk-neutral (Subsection 3.3.1) before considering the setting with a risk-averse agent (Subsection 3.3.2).

\subsubsection{Disclosure Value with a Two-task Risk-neutral Agent}

With a risk-neutral agent, the principal's objective is to motivate an efficient effort allocation across tasks. Note that if the gross payoff to the principal is non-contractible information, then risk neutrality is not sufficient to achieve the first-best result (Feltham and Xie 1994). Moreover, with both information systems $\eta_{1}$ and $\eta_{2}$, given non-contractible performance measures $y_{1}$ and $y_{2}$, market price is the sole contractible performance measure.

Let $\pi\left(\eta_{k}\right)=\mu_{k 1} a_{1}+\mu_{k 2} a_{2}+\varepsilon_{k \pi}$ represent market price given information system $\eta_{k}, k=1,2$, where the sensitivity to the agent's actions (i.e., $\mu_{k 1}$ and $\mu_{k 2}$ ) and the noise in price (i.e., $\mathcal{E}_{k \pi}$ ) all depend on $\eta_{k}$. More specifically, the parameters depend on the aggregation weights implied by $\eta_{k}$. Using this parameterization in (7b), assuming the agent is risk-neutral (i.e., $r \rightarrow 0$ ), yields the principal's expected net payoff, given by

$$
\Pi^{\dagger}\left(\eta_{k}\right)=1 / 2 Q_{k}^{-1}\left(b_{1} \mu_{k 1}+b_{2} \mu_{k 2}\right)^{2}, \quad k=1,2,
$$

with $Q_{k} \equiv \mu_{k 1}{ }^{2}+\mu_{k 2}{ }^{2}$. Substituting (13) for $k=1,2$ into (8) and simplifying yields the value of the additional, publicly reported performance measure $y_{2}$, i.e.,

$$
V^{\dagger}\left(y_{2} \mid \eta_{1}\right)=1 / 2 Q_{1}^{-1} Q_{2}^{-1} A_{12}\left[A_{b 1}\left(b_{1} \mu_{21}+b_{2} \mu_{22}\right)+A_{b 2}\left(b_{1} \mu_{11}+b_{2} \mu_{12}\right)\right],
$$

where $A_{b k} \equiv b_{1} \mu_{k 2}-b_{2} \mu_{k 1}$ is the measure of non-congruity for market price $\pi\left(\eta_{k}\right)$, relative to the firm's terminal payoff, and $A_{12} \equiv \mu_{12} \mu_{21}-\mu_{11} \mu_{22}$ is the measure of alignment between market prices $\pi\left(\eta_{1}\right)$ and $\pi\left(\eta_{2}\right)$ as implied by information system $\eta_{k}$ (Feltham and $\left.\mathrm{Wu} 2000\right)$.

Disclosure value of $y_{2}$ depends in a non-trivial way on the measures of non-congruity for the two information systems. ${ }^{23}$ For example, if the market price that is induced by the initial performance measure is perfectly congruent (i.e., if $A_{b 1}=0$ ), (14) simplifies to $V^{\dagger}\left(y_{2} \mid \eta_{1}\right)=-1 / 2 Q_{2}^{-1} A_{b 2}{ }^{2} \leq 0$. Then, disclosing a supplemental performance measure that yields a non-congruent price (i.e., a signal with $A_{b 2} \neq 0$ ) is detrimental

\footnotetext{
${ }^{23}$ Note that the two measures of non-congruity and the measure of alignment are functionally related.
} 
to the principal. Key to this result is that risk neutrality and a perfectly congruent market price under $\eta_{1}$ are sufficient to achieve the first-best result. On the other hand, while disclosing a supplemental performance measure improves market price's informativeness regarding the firm's terminal payoff, the expected net payoff to the principal declines if this signal is not congruent. Secondly, disclosing a supplemental performance measure is of no value to the principal if it is perfectly aligned with the initial signal (i.e., if $A_{12}$ $=0$ ). Given two aligned public signals, market price's measure of non-congruity is identical to the noncongruity for either signal. With risk neutrality, however, price congruency is the single key driver of inefficiencies in the agency.

More generally, price congruency depends on the weights assigned to public information. Using the signals' weights under $\eta_{1}$ and $\eta_{2}$ (Table 1,(12a), and (12b)) in (1a) yields a functional relation between market price, the agent's actions, and payoff and measurement noise. Table 2 summarizes these relations for $\eta_{1}$ and $\eta_{2}$ along with expressions for the price sensitivity to the agent's actions.

----- Insert Table 2 About Here. -----

Substituting (T2.1b), (T2.2b), and (T2.2c) into (14) yields a non-trivial expression for the value of the additional, publicly reported performance measure $y_{2}$. The sign of $V^{\dagger}\left(y_{2} \mid \eta_{1}\right)$ is, in general, ambiguous. Assuming that $y_{2}$ is used by investors in pricing the firm's terminal payoff(i.e., assuming that Lemma 2 does not apply), while disclosing supplemental information $y_{2}$ can be beneficial to the principal, for emphasis, we focus on conditions under which the disclosure value will be negative:

Proposition 4: Suppose the agent is risk-neutral; an additional, publicly reported performance measure has a negative disclosure value, for example, if:

(i) the initial performance measure is congruent with the firm's terminal payoff (i.e., $b_{1} / b_{2}=m_{11} / m_{12}$ );

(ii) one of the agent's tasks is window dressing that only affects the supplemental performance measure (i.e., $b_{i}=m_{1 i}=0$ and $m_{2 i} \neq 0$ for either $i=1$ or $i=2$ );

(iii) measurement error is unrelated, the supplemental performance measure covaries positively with the firm's terminal payoff and responds relatively more strongly to effort in the second task than does the initial performance measure, which, in turn, responds relatively more strongly to effort in the 
second task than does the firm's terminal payoff (i.e., $\rho=0, \rho_{2 x}>0$, and $m_{21} / m_{22}<m_{11} / m_{12}<b_{1} / b_{2}$ ).

Key to the negative disclosure value is the effect of disclosing $y_{2}$ on the congruency of market price, relative to the firm's terminal payoff. In particular, Proposition 4 identifies sufficient conditions under which disclosure of both, the initial plus the supplemental performance measure yields a less congruent price than does disclosure of merely the initial performance measure. For example, this holds in Proposition 4 (i) with a perfectly congruent initial performance measure, implying a likewise perfectly congruent market price. As a corollary, disclosure value will be negative if the initial performance measure is perfectly congruent in the sense that it does not respond to window dressing (Proposition 4 (ii)). Finally, Proposition 4 (iii) gives an example where the initial performance measure is non-congruent (e.g., it responds relatively more strongly to effort in the second task than does the firm's terminal payoff), but the supplemental performance measure is even less congruent (e.g., the relative response to effort in the second task is even more pronounced as compared with the initial signal). Here, any (positive) weighting of the two signals in the investors' pricing yields a less congruent market price than the weighting of merely the initial performance measure.

Note that the latter result is in stark contrast to the result for a two-task setting with contractible performance measures. There, releasing a non-aligned second performance measure is sufficient to achieve the first-best result. While in the latter setting it is the principal who selects the relative weights for the performance measures, in our setting it is the investors that weight the signals via their pricing. In particular, with contractible performance measures, the principal selects a negative weight for the supplemental performance measure (yielding a perfectly congruent total performance measure), while, due to the positive covariance between the supplemental performance measure and the firm's terminal payoff, the investors choose a positive weight.

\subsubsection{Disclosure Value with a Two-task Risk-averse Agent}

We now consider a simple model that integrates both reasons for a negative disclosure value, i.e., the market price is either noisier or less congruent as a consequence of the disclosure of a supplemental, non-contractible performance measure. The agent controls two tasks and there are two aggregate, non-contractible performance measures. Hence, the model is the same as the model in the previous subsection, except that the 
agent is risk averse. For illustration, we assume that the initial performance measure is perfectly congruent (i.e., $A_{b 1}=b_{1} m_{12}-b_{2} m_{11}=0$ ), the supplemental performance measure, in general, responds differently to the second task (i.e., $m_{21}=b_{1}$ and $m_{22}>0$ ), the noise variables have unit variance (i.e., $\sigma_{x}^{2}=\sigma_{\mu 1}{ }^{2}=\sigma_{\mu 2}{ }^{2}=1$ ), and measurement error is unrelated (i.e., $\rho=0$ ).

Proposition 5 characterizes two settings under which disclosing supplemental information $y_{2}$ will be detrimental to the principal.

Proposition 5: Suppose the agent is risk-averse; an additional, publicly reported performance measure has a negative disclosure value, for example, if:

(i) the agent's productivity is identical across tasks, the supplemental performance measure responds more strongly to effort in the second task than does the initial measure, and both signals are equally but limitedly informative regarding the firm's terminal payoff (i.e., $b_{1}=b_{2}=b, m_{22}>b, \rho_{1 x}=\rho_{2 x}=\rho_{x}$, and $\left.\rho_{x}^{2}<\underline{\rho}_{x}\right)$

(ii) the agent's second task is a window-dressing activity and the supplemental performance measure strongly responds to this activity (i.e., $b_{2}=0$ and $\left.m_{22}{ }^{2}>r \rho_{x 2}{ }^{-1}\left[2 \rho_{1 x}\left(1+\rho_{1 x}{ }^{2}\right)-\rho_{2 x}\left(\rho_{1 x}{ }^{2}+\rho_{2 x}{ }^{2}\right)\right]\right)$.

In the setting described by Proposition 5 (i), publicly reporting the supplemental performance measure results in a non-congruent price. However, given identical weights for both signals, market price's measure of non-congruity is unaffected by the payoff-informativeness of the two signals (i.e., $\rho_{x}$ ). On the contrary, price volatility is strictly monotonically increasing in payoff-informativeness. Thus, for a low informativeness, strong effort incentives (i.e., $v_{\pi}^{\dagger}$ ) result. Given that strong incentives amplify the consequences for the firm's terminal payoff of differences in the performance measure's congruity, a large downside of disclosing the supplemental signal exists, thus resulting in the negative disclosure value.

Likewise, in the setting of Proposition 5 (ii), price is non-congruent if supplemental information is publicly reported. On the other hand, depending on the signals' payoff-informativeness, disclosing both signals may yield a larger signal-to-noise ratio. While a non-congruent performance measure results in an inefficient effort allocation, the agent demands a risk premium to bear the performance measure inaccuracy. Therefore, disclosing supplemental information is detrimental to the principal if the loss in inefficient effort 
allocation exceeds the gains in risk premium. This is the case, if the supplemental performance measure strongly responds to the window-dressing activity.

\subsection{Disclosing Supplemental Contractible Information}

So far, the analysis addressed the value to the principal of supplemental, publicly reported non-contractible information. Subsequently, we will consider the value of disclosing contractible information in addition to other non-contractible information. Note that, following Proposition 2 (i), disclosing a single contractible performance measure has no value to the principal. Likewise, disclosing contractible information is, in general, of no value if the performance measure is publicly reported in addition to a single non-contractible performance measure. ${ }^{24}$ Key to this result is that the principal can always use the contractible performance measure to undo this signal's impact on market price; the "filtered" market price, however, is an equivalent statistic to market price in the setting where the contractible performance measure is not publicly reported. However, disclosing a contractible performance measure in addition to multiple non-contractible performance measures yields starkly different results.

To illustrate these differences, we extend the model in Subsection 3.2 by considering a contractible performance measure $y_{c}=m a+\rho_{c x} \mathcal{E}_{x}+\mathcal{E}_{\mu c}$ plus two non-contractible performance measures $y_{i}=m a+\rho_{i x} \mathcal{E}_{x}$ $+\mathcal{\varepsilon}_{\mu i}, i=1,2$, where $\mathcal{\varepsilon}_{\mu j} \sim \mathrm{N}\left(0, \sigma_{\mu j}^{2}\right), j=1,2, c, \operatorname{Cov}\left[\mathcal{\varepsilon}_{\mu j}, \mathcal{E}_{x}\right]=0, \operatorname{Cov}\left[\mathcal{E}_{\mu 1}, \mathcal{E}_{\mu 2}\right]=\rho \sigma_{\mu 1} \sigma_{\mu 2}$, and $\operatorname{Cov}\left[\mathcal{\varepsilon}_{\mu i}, \mathcal{\varepsilon}_{\mu c}\right]=\rho_{c i} \sigma_{\mu c} \sigma_{\mu i}$. While $\eta_{2}$ publicly reports both non-contractible performance measures (i.e., $y_{1}$ and $y_{2}$ ), $\eta_{3}$ also discloses the contractible performance measure (i.e., $\left.y_{c}\right)$. Thus, $V^{\dagger}\left(y_{c} \mid \eta_{2}\right)$ reflects the value of additional, publicly reported contractible information.

Similar to Subsection 3.2, using the model's parameterization in (1c), Lemma 3 summarizes conditions under which $y_{c}$ is not used in the price setting process.

Lemma 3: The capital market does not use supplemental information $y_{c}$ to set the price if

$$
\rho_{c x}=\left(1-\rho^{2}\right)^{-1}\left[\left(\rho_{c 2}-\rho \rho_{c 1}\right) \rho_{2 x} / \sigma_{\mu 2}+\left(\rho_{c 1}-\rho \rho_{c 2}\right) \rho_{1 x} / \sigma_{\mu 1}\right] \sigma_{\mu c} .
$$

This condition holds, for example, if:

\footnotetext{
${ }^{24}$ The only exception refers to the knife-edge case where the non-contractible information is no longer impounded in price, given the disclosure of the contractible information. Then, the principal is weakly worse off.
} 
(i) all three signals are not informative about the firm's terminal payoff (i.e., $\rho_{1 x}=\rho_{2 x}=\rho_{c x}=0$ );

(ii) the supplemental signal is not informative about either the firm's terminal payoff or the measurement error of the initial signals (i.e., $\rho_{c 1}=\rho_{c 2}=\rho_{c x}=0$ );

(iii) at least one non-contractible signal is informative about the firm's terminal payoff and contains no measurement error (i.e., $\rho_{i x} \neq 0$ and ${\sigma_{\mu i}}^{2}=0$ for $i=1$ or $i=2$ );

(iv) the non-contractible signals are identical (i.e., $\rho_{1 x}=\rho_{2 x}, \sigma_{\mu 1}=\sigma_{\mu 2}, \rho_{c 1}=\rho_{c 2}$, and $\rho=1$ ).

Observe the similarity between the conditions in Lemma 3 and those in Lemma 2. More specifically, the line of reasoning for Lemma 3 parallels the logic to Lemma 2. In particular, if any condition outlined in Lemma 3 holds, $y_{c}$ is not used to derive the firm's price and the value of disclosing $y_{c}$ is zero.

Note that information systems $\eta_{2}$ and $\eta_{3}$ are identical except for the induced market price. Thus, the performance comparison of $\eta_{2}$ and $\eta_{3}$ relies on the sensitivity of market price to the agent's action and price's (conditional) variance. Similar to Subsection 3.2, given that supplemental information is impounded in price, disclosure of $y_{c}$ (weakly) increases price volatility (i.e., $\sigma_{\pi}^{2}\left(\eta_{3}\right) \geq \sigma_{\pi}^{2}\left(\eta_{2}\right)$ if Lemma 3 does not hold). On the contrary, the impact of disclosing $y_{c}$ on both, the sensitivity of market price and the correlation between market price and the contractible performance measure, is ambiguous. Hence, in general, publicly reporting an additional contractible performance measure can be both beneficial and detrimental to the principal.

Applying the model's parameterization to (8), using (7e) and (7f), results in an expression for the disclosure value $V^{\dagger}\left(y_{c} \mid \eta_{2}\right)$. However, using this expression to identify cutoff-values yields rather complex closed-form solutions. Hence, we employ a numerical example to demonstrate that, with multiple noncontractible performance measures, the principal can have strict preferences regarding the disclosure of additional contractible information.

Figure 1 illustrates these preferences. Here, $m=b=1, r=1, \sigma_{\mu 1}=\sigma_{\mu 2}=\sigma_{\mu c}=\sigma_{x}=1, \rho_{1 x}=1 / 8, \rho_{2 x}=1 / 2$, $\rho_{c x}=\rho_{c 1}=\rho_{c 2}=1 / 10$.

------ Insert Figure 1 About Here.

In the example, besides three knife-edge conditions, the principal is not indifferent regarding the disclosure of contractible information. More specifically, the principal benefits from publicly reporting $y_{c}$ 
if measurement error of the two non-contractible performance measures is highly correlated (i.e., for large values of $|\rho|$ ). With $\eta_{2}$, for a relatively negative correlation, the investors assign a negative weight to one signal, yielding a weak sensitivity of market price to the agent's action. On the other hand, for a large positive correlation, a high volatility results. In both cases, disclosure of $y_{c}$ under $\eta_{3}$ increases the sensitivity of market price to the agent's action, which is particularly beneficial in case of a relatively negative correlation.

Notably, unlike disclosure of a contractible performance measure in settings with either no or merely a single publicly reported, non-contractible performance measure, the principal has strict preferences for disclosing contractible information in settings with multiple public, non-contractible performance measures. Then, the value of disclosing contractible information depends on the information already available to the capital market. Intuitively, disclosure affects the investors' inferences regarding the firm's terminal payoff, the weights that they assign to the disclosed performance measures and, as a consequence, the sensitivity and (conditional) variance of market price. As the numerical example illustrates, the performance comparison of disclosure versus non-disclosure can go either way. Proposition 6 summarizes necessary conditions for the principal to have a strict preference regarding the disclosure of an additional contractible performance measure.

Proposition 6: Suppose that information system $\eta_{s}$ is identical to information system $\eta_{o}$, except for the additional disclosure of a contractible performance measure $y_{c}$; necessary conditions for the principal to have a strict preference regarding the disclosure or non-disclosure of a contractible performance measure (i.e., $\left.V^{\dagger}\left(y_{c} \mid \eta_{o}\right) \neq 0\right)$ include,

(i) $\eta_{o}$ publicly reports at least two non-contractible performance measures;

(ii) disclosure of $y_{c}$ alters investors' aggregation of information into price (i.e., $\alpha_{c}^{*}\left(\eta_{s}\right) \neq 0, \alpha_{i}^{*}\left(\eta_{s}\right) \neq \alpha_{i}^{*}\left(\eta_{o}\right)$ for at least one $i \in \mathbf{Y}_{o}$ );

(iii) with $\eta_{o}$, the principal uses market price $\pi$ in the contract with the agent (i.e., with $\eta_{o}, v_{\pi}{ }^{*} \neq 0$ ).

Note that Proposition 6 carries forward to a multi-task setting. There, altered weights imply a (weak) change in the market price's congruency with the firm's terminal payoff. Similarly, the change in congruency 
can be beneficial as well as detrimental to the principal. For example, suppose agent risk-aversion is negligible. Then, the value of disclosure will be negative if the contractible performance measure and price are not aligned with $\eta_{o}$, but they are aligned with $\eta_{s}$.

As a corollary to Proposition 6 , if the new information is price relevant (i.e., $\alpha_{c}^{*}\left(\eta_{s}\right) \neq 0$ ), the principal will have strict preferences regarding its disclosure. However, while price informativeness weakly increases as a consequence of the additional information that is available to investors, disclosing contractible information can leave the principal strictly worse off.

\section{CONCLUding Remarks}

In this paper, we examine the economic consequences of disclosing different types of information to the capital market. Our study is motivated by the recently proposed focus on decision-usefulness as the primary objective of financial accounting and the ongoing demand for more disclosure of soft information. In our analysis, market price serves as a performance measure in the contract between a risk neutral principal and a risk and effort averse agent. We extend a standard multi-task LEN-model to include the firm's market price and investigate the relation between price efficiency and solution of an agency conflict. More specifically, we investigate the disclosure of additional soft and hard information from a stewardship perspective.

We find that while publicly reported additional information improves price efficiency, the impact of more disclosed information on the principal's expected net payoff is ambiguous. Hence, we present a rationale for partial disclosure that is complementary to proprietary costs or uncertainty concerning the quantity of information. Contrary to much of the earlier work on partial disclosure, rather than withholding information ex post because it draws a bad picture of the firm (and thus leads to a lower stock-price), the principal commits ex ante (i.e., before the information is generated) to not disclose the information. In our setting, withholding information is valuable because the contracting usefulness of market price decreases as a consequence of the disclosure.

Based on our findings, we conclude that stewardship and decision-usefulness are potentially conflicting 
objectives of financial accounting. This is consistent, e.g., with empirical evidence provided by Gassen (2008). Thus, abandoning stewardship as a separate objective of financial accounting and mandating further disclosure of soft information by standard setters and regulators can result in lower productivity and reduced firm value. Also, firms can be compelled to offset the negative consequences of mandatory disclosure by reducing their amount of voluntary disclosure. Likewise, it can be optimal for firms to disclose aggregated soft information (if possible) instead of detailed information. This result is similar to Bushman and Indjejikian (1993), who show that it can be optimal to disclose distorted information.

Our findings have implications for empiricists, standard setters, and regulators. Given that noncontractible information is not verifiable and, thus, prone to window dressing by managers, disclosing (more) soft information will result in a re-allocation of effort from productive to non-productive tasks. Moreover, increasing the level of disclosure yields a noisier price. Hence, assuming a mandatory disclosure regime, we expect to observe a larger relative weight on accounting earnings as compared to stock price after more comprehensive requirements for disclosure of soft information are in effect. In a similar vain, increased mandatory requirements can result in a reduction of the amount of voluntary disclosure, including the disclosure of hard information. Finally, standard setters frequently change disclosure standards one at a time. Our results, however, indicate that the "value of a standard" crucially depends on the standards that are already in place. In general, adjusting a standard in the sense of increasing demand for disclosing either hard or soft information can destroy firm value. 


\section{$-30-$ \\ APPENDIX A \\ PROOF OF PROPOSITIONS}

Proposition 1: The principal's unconstrained decision problem is given by (6). (i) Setting $\mathbf{v}_{c}=\mathbf{0}$ and differentiating (6) with respect to $v_{\pi}$ and solving the first order condition for $v_{\pi}$ gives (7a). Substituting (7a) and $\mathbf{v}_{c}=\mathbf{0}$ into (6) yields the principal's expected net payoff as characterized by (7b). (ii) Setting $v_{\pi}=0$ and differentiating (6) with respect to $\mathbf{v}_{c}$ and solving the first order conditions for all $\mathbf{v}_{c}$ yields (7c). Substituting (7c) plus $v_{\pi}=0$ into (6) and simplifying yields the principal's expected net payoff as depicted in (7d). (iii) Differentiating (6) with respect to $v_{\pi}$ and $\mathbf{v}_{c}$, and solving for $v_{\pi}$ and all $\mathbf{v}$ yields (7e). Substituting (7e) in (6) yields the principal's expected net payoff as characterized by (7f).

Lemma 2: Applying the model's parameterization to (1c) yields the following weights that are assigned to the two non-contractible signals by the investors:

$$
\begin{aligned}
& \alpha_{1}{ }^{*}=Q^{-1}\left(\rho_{1 x} \sigma_{\mu 2}-\rho \rho_{2 x} \sigma_{\mu 1}\right) \sigma_{\mu 2} \sigma_{x}^{2}, \\
& \alpha_{2}{ }^{*}=Q^{-1}\left(\rho_{2 x} \sigma_{\mu 1}-\rho \rho_{1 x} \sigma_{\mu 2}\right) \sigma_{\mu 1} \sigma_{x}^{2},
\end{aligned}
$$

where $Q \equiv\left(\rho_{1 x}{ }^{2} \sigma_{x}{ }^{2}+\sigma_{\mu 1}{ }^{2}\right)\left(\rho_{2 x}{ }^{2} \sigma_{x}{ }^{2}+\sigma_{\mu 2}{ }^{2}\right)-\left(\rho_{1 x} \rho_{2 x} \sigma_{x}{ }^{2}+\rho \sigma_{\mu 1} \sigma_{\mu 2}\right)^{2}$. Following (A.1b), the weight assigned to the supplemental information $y_{2}$ will be zero if $\left(\rho_{2 x} \sigma_{\mu 1}-\rho \rho_{1 x} \sigma_{\mu 2}\right) \sigma_{\mu 1}=0$. Settings (i) to (iv) describe conditions under which the weight will be zero.

Proposition 3: Substituting (9a), (9b), and $\rho_{1 x}=\rho_{2 x}=\rho_{x}$ into (11) and simplifying yields the value of disclosing $y_{2}$, given disclosure of $y_{1}$ as

$$
V^{\dagger}\left(y_{2} \mid \eta_{1}\right)=1 / 2 \frac{b^{2} m^{2} r \sigma_{\mu 1}^{2}\left(\sigma_{\mu 1}-\rho \sigma_{\mu 2}\right)^{2}}{\left[m^{2}+r\left(\sigma_{\mu 1}^{2}+\rho_{x}^{2} \sigma_{x}^{2}\right)\right]\left[\left(m^{2}\left(\sigma_{\mu 1}^{2}-2 \rho \sigma_{\mu 1} \sigma_{\mu 2}+\sigma_{\mu 2}^{2}\right)+r Q\right]\right.} \geq 0,
$$

with $Q \equiv\left(\rho_{x}^{2} \sigma_{x}^{2}+\sigma_{\mu 1}{ }^{2}\right)\left(\rho_{x}^{2} \sigma_{x}^{2}+\sigma_{\mu 2}{ }^{2}\right)-\left(\rho_{x}^{2} \sigma_{x}^{2}+\rho \sigma_{\mu 1} \sigma_{\mu 2}\right)^{2}$. Since $V^{\dagger}\left(y_{2} \mid \eta_{1}\right) \geq 0, \rho_{1 x} \neq \rho_{2 x}$ is a necessary condition. (i) Substituting (9a), (9b), and $\sigma_{\mu 2}=0$ into (11) and simplifying yields

$$
V^{\dagger}\left(y_{2} \mid \eta_{1}\right)=1 / 2 \frac{b^{2} m^{2} r\left[\sigma_{\mu 1}+\left(\rho_{1 x}^{2}-\rho_{2 x}^{2}\right) \sigma_{x}^{2}\right]}{\left[m^{2}+r \rho_{2 x}^{2} \sigma_{x}^{2}\right]\left[m^{2}+r\left(\sigma_{\mu 1}^{2}+\rho_{1 x}^{2} \sigma_{x}^{2}\right)\right]} .
$$


The numerator in (A.2) is negative if $\sigma_{\mu 1}+\left(\rho_{1 x}{ }^{2}-\rho_{2 x}{ }^{2}\right) \sigma_{x}{ }^{2}<0$. Solving this condition for $\rho_{2 x}{ }^{2}$ yields the cutoff value for the payoff-informativeness of the second performance measure. Since $\rho_{2 x} \in[-1,1]$, this cutoff value must be strictly smaller than 1 for a non-empty solution set. Solving the latter condition for $\sigma_{\mu 1}{ }^{2}$ results in the cutoff value for the measurement error of the first performance measure. (ii) Substituting (9a), (9b), and $\sigma_{\mu 2}$ $=0$ into (11) and simplifying yields

$$
V^{\dagger}\left(y_{2} \mid \eta_{1}\right)=1 / 2 \frac{b^{2} m^{2} r \rho_{2 x} \sigma_{\mu 1}^{2}\left[\rho_{2 x} \sigma_{\mu 1}^{2}\left(\sigma_{\mu 1}^{2}+\sigma_{\mu 2}^{2}\right)+\left(\rho_{1 x}-\rho_{2 x}\right)^{2} \rho_{2 x} \sigma_{\mu 1}^{2} \sigma_{x}^{2}+2\left(\rho_{1 x}-\rho_{2 x}\right) Q\right]}{\left[m^{2}+r\left(\sigma_{\mu 1}^{2}+\rho_{1 x}^{2} \sigma_{x}^{2}\right)\right]\left[m^{2}\left(\rho_{1 x} \sigma_{\mu 2}^{2}+\rho_{2 x} \sigma_{1 x}^{2}\right)^{2}+r\left(\rho_{1 x}^{2} \sigma_{\mu 2}^{2}+\rho_{2 x}^{2} \sigma_{\mu 1}^{2}\right) Q\right]},
$$

where $Q=\rho_{1 x}{ }^{2}{\sigma_{\mu 2}}^{2}{\sigma_{x}}^{2}+{\sigma_{\mu 1}}^{2}\left({\sigma_{\mu 2}}^{2}+\rho_{2 x}{ }^{2} \sigma_{x}{ }^{2}\right)$. The denominator in (A.3) is positive; the numerator is negative if

$$
\sigma_{1}^{2}\left[2 \rho_{1 x} \sigma_{\mu 2}^{2}+\rho_{2 x}\left(\sigma_{\mu 1}^{2}-{\sigma_{\mu 2}}^{2}+\left(\rho_{1 x}{ }^{2}-\rho_{2 x}{ }^{2}\right) \sigma_{x}^{2}\right)\right]<2 \rho_{1 x}{ }^{2}\left(\rho_{2 x}-\rho_{1 x}\right) \sigma_{\mu 2}{ }^{2} \sigma_{x}^{2}
$$

For $\rho_{2 x}>\rho_{1 x}>0$, the right hand side of (A.4) is positive. Thus, the numerator is negative if the left hand side of (A.4) is negative, i.e.,

$$
2 \rho_{1 x} \sigma_{\mu 2}^{2}+\rho_{2 x}\left(\sigma_{\mu 1}^{2}-\sigma_{\mu 2}^{2}+\left(\rho_{1 x}^{2}-\rho_{2 x}^{2}\right) \sigma_{x}^{2}\right)<0 .
$$

Solving the latter condition for ${\sigma_{\mu 1}}^{2}$ yields the cutoff value for the measurement error of the first performance measure. Finally, for $\rho_{2 x}>2 \rho_{1 x}$ this cutoff value is positive, yielding a non-empty solution set.

Propositions 4: (i) First, substitute (T2.1b), (T2.2b), and (T2.2c) into (14) to obtain an expression for the value of the additional, publicly reported performance measure. (i) Substituting $m_{11} / m_{12}=b_{1} / b_{2}$ yields

$$
V^{\dagger}\left(y_{2} \mid \eta_{1}\right)=-1 / 2 Q^{-1} b_{1}^{2}\left(b_{1} m_{22}-b_{2} m_{21}\right)^{2} \sigma_{\mu 1}^{2}\left(\rho_{2 x} \sigma_{\mu 1}-\rho \rho_{1 x} \sigma_{\mu 2}\right)^{2} \leq 0 .
$$

Interestingly, the value is zero, e.g., if the second performance measure is perfectly congruent with the firm's terminal payoff (i.e., $b_{1} / b_{2}=m_{21} / m_{22}$ ) and if the second performance measure is not impounded in the price (i.e., $\rho_{2 x} \sigma_{\mu 1}-\rho \rho_{1 x} \sigma_{\mu 2}=0$, see Lemma 2). (ii) For example, substituting $b_{2}=m_{12}=0$ into the expression for disclosure value yields

$$
V^{\dagger}\left(y_{2} \mid \eta_{1}\right)=-1 / 2 \frac{m_{22}^{2} b_{1}^{2} \sigma_{\mu 1}^{2}\left(\rho_{2 x} \sigma_{\mu 1}-\rho \rho_{1 x} \sigma_{\mu 2}\right)^{2}}{m_{22} \sigma_{\mu 1}^{2}\left(\rho_{2 x} \sigma_{\mu 1}-\rho \rho_{1 x} \sigma_{\mu 2}\right)^{2}+\left[m_{11} \sigma_{\mu 2}\left(\rho_{1 x} \sigma_{\mu 2}-\rho \rho_{2 x} \sigma_{\mu 1}\right)+m_{21} \sigma_{\mu 1}\left(\rho_{2 x} \sigma_{\mu 1}-\rho \rho_{1 x} \sigma_{\mu 2}\right)\right]^{2}}
$$

which is non-positive. In particular, disclosure value will be negative if the supplemental information is impounded in the price. (iii) Substituting $\rho=0$ into the expression for disclosure value yields 


$$
\begin{aligned}
& V^{\dagger}\left(y_{2} \mid \eta_{1}\right)= \\
& -1 / 2 \frac{\Lambda_{12} \rho_{2 x} \sigma_{\mu 1}^{2}\left[\left(b_{1}\left(m_{21} \Lambda_{b 1}+m_{11} \Lambda_{b 2}\right)+b_{2}\left(m_{22} \Lambda_{b 1}+m_{12} \Lambda_{b 2}\right)\right) \rho_{2 x} \sigma_{\mu 1}^{2}+2\left(b_{1} m_{11}+b_{2} m_{12}\right) \Lambda_{b 1} \rho_{1 x} \sigma_{\mu 2}^{2}\right]}{\left(m_{11}^{2}+m_{12}^{2}\right)\left[\left(m_{21}^{2}+m_{22}^{2}\right) \rho_{2 x}^{2} \sigma_{\mu 1}^{4}+2\left(m_{11} m_{21}+m_{12} m_{22}\right) \rho_{1 x} \rho_{2 x} \sigma_{\mu 1}^{2} \sigma_{\mu 2}^{2}+\left(m_{11}^{2}+m_{12}^{2}\right) \rho_{1 x}^{2} \sigma_{\mu 2}^{4}\right]},
\end{aligned}
$$

where $\Lambda_{12}=m_{11} m_{22}-m_{12} m_{21}, \Lambda_{b 1}=b_{1} m_{12}-b_{2} m_{11}$, and $\Lambda_{b 2}=b_{1} m_{22}-b_{2} m_{21}$ are the measures of alignment and non-congruity, respectively, for the non-contractible performance measures. Disclosure value will be negative if $\Lambda_{12}>0, \Lambda_{b 1}>0, \Lambda_{b 2}>0$, and $\rho_{2 x}>0$. Evaluation of the conditions for the measures of alignment and non-congruity yields the relation of the sensitivities of the performance measures and the tasks' productivity.

Propositions 5: Substituting the model's parameterization into ( $7 b$ ) and (8) yields an expression for the value of the additional, publicly reported performance measure. (i) Substituting $b_{1}=b_{2}=b$ and $\rho_{1 x}=\rho_{2 x}=\rho_{x}$ results in the expression for the disclosure value for this setting. The disclosure value is negative if

$$
\rho_{x}^{2}<\varrho_{x} \equiv \frac{2 b^{2}\left(b-m_{22}\right)^{2}-r\left[\left(b+m_{22}\right)^{2}+4 b m_{22}\right]}{r\left[4 b\left(-2 b+m_{22}\right)+\left(b+m_{22}\right)^{2}\right]},
$$

where $\rho_{x}=\underline{\rho}_{x}$ yields $V^{\dagger}\left(y_{2} \mid \eta_{1}\right)=0$. Finally, $m_{22}>b$ ensures that the numerator of the above expression is positive. (ii) Substituting $b_{2}=0$ in the expression for the disclosure value, setting the numerator equal to zero and solving the equation for $m_{22}^{2}$ yields

$$
\underline{m}_{22}^{2} \equiv m_{22}^{2}=r \rho_{x 2}^{-1}\left[2 \rho_{1 x}\left(1+\rho_{1 x}^{2}\right)-\rho_{2 x}\left(\rho_{1 x}^{2}+\rho_{2 x}^{2}\right)\right] .
$$

The slope of $V^{\dagger}\left(y_{1} \mid \eta_{2}\right)$ at $m_{22}{ }^{2} \equiv \underline{m}_{22}{ }^{2}$ is negative; thus, disclosure value is negative if $m_{22}{ }^{2}>\underline{m}_{22}{ }^{2}$.

Lemma 3: Applying the model's parameterization to (1c) yields the following weights that are assigned to the contractible signal $y_{c}$ and the two non-contractible signals $y_{i}, i=1,2$ by the investors:

$$
\begin{aligned}
& \alpha_{c}^{*}=Q^{-1}\left[\left(1-\rho^{2}\right) \rho_{c x} \sigma_{\mu 1} \sigma_{\mu 2}-\left(\left(\rho_{c 1}-\rho \rho_{c 2}\right) \rho_{1 x} \sigma_{\mu 2}+\left(\rho_{c 2}-\rho \rho_{c 1}\right) \rho_{2 x} \sigma_{\mu 1}\right) \sigma_{c}\right] \sigma_{\mu 1} \sigma_{\mu 2} \sigma_{x}^{2}, \\
& \alpha_{1}^{*}=Q^{-1}\left[\left(1-\rho_{c 2}{ }^{2}\right) \rho_{1 x} \sigma_{\mu 2} \sigma_{c}-\left(\left(\rho_{c 1}-\rho \rho_{c 2}\right) \rho_{c x} \sigma_{\mu 2}+\left(\rho-\rho_{c 1} \rho_{c 2}\right) \rho_{2 x} \sigma_{c}\right) \sigma_{\mu 1}\right] \sigma_{\mu 2} \sigma_{c} \sigma_{x}^{2}, \\
& \alpha_{2}^{*}=Q^{-1}\left[\left(1-\rho_{c 1}{ }^{2}\right) \rho_{2 x} \sigma_{\mu 1} \sigma_{c}-\left(\left(\rho_{c 2}-\rho \rho_{c 1}\right) \rho_{c x} \sigma_{\mu 1}+\left(\rho-\rho_{c 1} \rho_{c 2}\right) \rho_{1 x} \sigma_{c}\right) \sigma_{\mu 2}\right] \sigma_{\mu 1} \sigma_{c} \sigma_{x}^{2}
\end{aligned}
$$


where $Q$ is the determinant of $\boldsymbol{\Sigma}_{p}$. Following (A.6a), the weight assigned to the supplemental contractible information $y_{c}$ will be zero if $\left(1-\rho^{2}\right) \rho_{c x} \sigma_{\mu 1} \sigma_{\mu 2}-\left(\left(\rho_{c 1}-\rho \rho_{c 2}\right) \rho_{1 x} \sigma_{\mu 2}+\left(\rho_{c 2}-\rho \rho_{c 1}\right) \rho_{2 x} \sigma_{\mu 1}\right) \sigma_{c}=0$. Settings (i) to (iv) describe conditions under which the weight will be zero.

Proposition 6: Proof is by contradiction. (i) If there is only a single publicly reported non-contractible signal, by subtracting appropriately weighted contractible performance measures, the principal obtains a filtered market price that only reflects the non-contractible information. This result holds for an arbitrary number of disclosed contractible performance measures. Therefore, disclosure does not change the characteristics of the set of contractible performance measures. (ii) Suppose that disclosure does not affect investors' aggregation of information into price. Then, the principal can filter out the impact of the additional, contractible information from market price and obtains a filtered price that is an equivalent statistic to the initial market price where the supplemental information is not disclosed. (iii) If the principal does not initially use market price in the contract with the agent, disclosure will not deteriorate the characteristics of the set of contractible performance measures. Here, disclosure value is non-negative. 


\section{REFERENCES}

Baiman, S. and R.E. Verrecchia (1995) "Earnings and Price-based Compensation Contracts in the Presence of Discretionary Trading and Incomplete Contracting," Journal of Accounting and Economics 20, 93-121.

Bushman, R., E. Engel, and A. Smith (2006) "An Analysis of the Relation between the Stewardship and Valuation Roles of Earnings," Journal of Accounting Research 44, 53-83.

Bushman, R.M. and R.J. Indjejikian (1993) "Stewardship Value of 'Distorted' Accounting Disclosures," Accounting Review 68, 765-782.

Chen, X., Q. Cheng, and K. Lo (2006) “Are Analyst Research and Corporate Disclosures Complements or Substitutes?" working paper.

Choi, J.P., E.G. Kristiansen, and J. Nahm (2007) “Communication of Soft Information to Lenders: Credibility and Reputation," working paper.

Christensen, P.O. and G.A. Feltham (2000) "Market Based Performance Measures and Disclosure of Private Management Information in Capital Markets," Review of Accounting Studies 5, 301-329.

Core, J.E., W.R. Guay, and R.E. Verrecchia (2003) "Price vs. Non-Price Performance Measures in Optimal CEO Compensation Contracts," Accounting Review 78, 957-981.

Davis, A., J. Piger, and L. Sedor (2007) "Beyond the Numbers: Managers' Use of Optimistic and Pessimistic Tone in Earnings Press Releases," working paper.

Demers, E. and C. Vega (2008) “Soft Information in Earnings Announcements: News or Noise?" working paper.

Dye, R.A. (1985) “Disclosure of Nonproprietary Information,” Journal of Accounting Research 23, 123-145.

Feltham, G.A. and J. Xie (1994) "Performance Measure Congruity and Diversity in Multi-Task Principal/Agent Relations," Accounting Review 69, 429-453.

Feltham, G.A. and M.G.H. Wu (2000) "Public Reports, Information Acquisition by Investors, and Management Incentives," Review of Accounting Studies 5, 155-190.

Fischer, P. and R. Verrecchia (2000) “Reporting Bias,” Accounting Review 75, 229-245.

Gassen, J. (2008) "Are Stewardship and Valuation Usefulness Compatible or Alternative Objectives of Financial Accounting?" working paper.

Gjesdal, F. (1981) “Accounting for Stewardship,” Journal of Accounting Research 19, 208-231.

Guay, W. and R.E. Verrecchia (2007) “Conservative Disclosure,” working paper.

Hayes, R.M. and S. Schaefer (2000) "Implicit Contracts and the Explanatory Power of Top Executive Compensation for Future Performance," RAND Journal of Economics 31, 273-293.

Healy, P.M. and K.G. Palepu (2001) "Information Asymmetry, Corporate Disclosure, and the Capital Markets: A Review of the Empirical Disclosure Literature," in Journal of Accounting and Economics 31, 405-440. 
International Accounting Standards Board - IASB (2005) “Agenda Paper 8A, 19 October 2005, Information for Observers," available at http://www.iasb.org/NR/rdonlyres/C936AA1E-FE3A-4A7F-BE06-60644C9DA7E6/0/Oct050510o b08a.pdf.

Kim, S.K. and Y.S. Suh (1991) "Ranking of Accounting Information Systems for Management Control," Journal of Accounting Research 29, 386-396.

Kyle, A.S. (1985) “Continuous Auctions and Insider Trading,” Econometrica 53, 1315-1335.

Lambert, R.A. (2001) “Contracting Theory and Accounting," Journal of Accounting and Economics 32, 3-87.

Leone, A.J., J.S. Wu, and J.L. Zimmerman (2006) "Asymmetric Sensitivity of CEO Cash Compensation to Stock Returns," Journal of Accounting and Economics 42, 167-192.

Liberti, J.M. (2003) "Initiative, Incentives and Soft Information How Does Delegation Impact the Role of Bank Relationship Managers?” working paper.

O’Connell, V. (2007) “Reflections on Stewardship Reporting," Accounting Horizons 21, 215-227.

Paul, J.M. (1992) “On the Efficiency of Stock-based Compensation,” Review of Financial Studies 5, 471-502.

Petersen, M.A. (2004) “Information: Hard and Soft," working paper.

Seamons, Q.F. and R.W. Rouse (1997) “How to Use Soft Harbor to Report 'Soft' Information," Journal of Corporate Accounting and Finance 9, 81-90.

Stocken, P.C. and R.E. Verrecchia (1999) "Performance Monitoring and Financial Disclosure Choice," Journal of Institutional and Theoretical Economics 155, 214-232.

Teoh, S.H. and C.Y. Kwan (1991) “Nondisclosure and Adverse Disclosure as Signals of Firm Value,” Review of Financial Studies 4, 283-313.

Tetlock, P.C., M. Saar-Tsechansky, and S. Macskassy (2008) "More Than Words: Quantifying Language to Measure Firms' Fundamentals,” Journal of Finance 63, 1437 - 1467.

Tsao, A. (2002) “When a Stock’s Rating and Target Collide,” BusinessWeek Online.

Verrecchia, R.E. (1983) “Discretionary Disclosure," Journal of Accounting and Economics 5, 179-194.

Verrecchia, R.E. (2001) “Essays on Disclosure,” Journal of Accounting and Economics 32, 97-180. 


\section{Table 1}

\section{Disclosure of a Single Performance Measure}

\section{Panel A: Performance Measure $y_{1}$ is Hard Information}

$\eta_{0}$ - no public performance measure

$$
\begin{aligned}
& a^{\dagger}\left(v_{1}\right)=m v_{1}, \\
& \pi\left(\eta_{0}\right)=\left(m^{2}+r \sigma_{1}^{2}\right)^{-1} b^{2} m^{2}, \\
& v_{1}^{\dagger}=\left(m^{2}+r \sigma_{1}^{2}\right)^{-1} b m, \\
& \Pi^{\dagger}\left(\eta_{0}\right)=1 / 2\left(m^{2}+r \sigma_{1}^{2}\right)^{-1} b^{2} m^{2},
\end{aligned}
$$

$\eta_{1}$ - disclosure of $y_{1}$

$$
\begin{aligned}
& a^{\dagger}\left(v_{1}, v_{\pi}\right)=m v_{1}+\alpha^{*} m v_{\pi}, \\
& \pi\left(y_{1} \mid \eta_{1}\right)=\left(m^{2}+r \sigma_{1}^{2}\right)^{-1} b^{2} m^{2}+\alpha^{*}\left(y_{1}-\mathrm{E}\left[y_{1} \mid \hat{a}, z, \eta_{1}\right]\right), \\
& v_{1}^{\dagger}=\left(m^{2}+r \sigma_{1}^{2}\right)^{-1} b m \quad \text { and } \quad v_{\pi}^{\dagger}=0, \\
& \Pi^{\dagger}\left(\eta_{1}\right)=1 / 2\left(m^{2}+r \sigma_{1}^{2}\right)^{-1} b^{2} m^{2},
\end{aligned}
$$

with $\quad \alpha^{*}=\left(\rho_{1 x}{ }^{2}+\sigma_{\mu 1}{ }^{2} / \sigma_{x}^{2}\right)^{-1} \rho_{1 x}$,

$\sigma_{1}^{2}=\rho_{1 x}^{2} \sigma_{x}^{2}+\sigma_{\mu 1}^{2}$, and

$\mathrm{E}\left[y_{1} \mid \hat{a}, z, \eta_{1}\right]=\left(m^{2}+r \sigma_{1}^{2}\right)^{-1} b m^{3}$.

\section{Panel B: Performance Measure $y_{1}$ is Soft Information}

$\eta_{0}$ - no public performance measure

$$
\begin{aligned}
& a^{\dagger}=0, \\
& \pi\left(\eta_{0}\right)=b \hat{a}=0, \\
& v_{1}^{\dagger}=0, \\
& \Pi^{\dagger}\left(\eta_{0}\right)=0,
\end{aligned}
$$

$\eta_{1}$ - disclosure of $y_{1}$ with $\rho_{1 x} \neq 0$

$$
\begin{aligned}
& a^{\dagger}\left(v_{\pi}\right)=\alpha^{*} m v_{\pi}, \\
& \pi\left(y_{1} \mid \eta_{1}\right)=\left(m^{2}+r \sigma_{\pi}^{2} / \alpha^{* 2}\right)^{-1} b^{2} m^{2}+\alpha^{*}\left(y_{1}-\mathrm{E}\left[y_{1} \mid \hat{a}, z, \eta_{1}\right]\right), \\
& v_{1}^{\dagger}=0 \quad \text { and } \quad v_{\pi}^{\dagger}=1 / \alpha^{*}\left(m^{2}+r \sigma_{\pi}^{2} / \alpha^{* 2}\right)^{-1} b m, \\
& \Pi^{\dagger}\left(\eta_{1}\right)=1 / 2\left[m^{2}+r\left(\rho_{1 x}{ }^{2} \sigma_{x}^{2}+\sigma_{\mu 1}{ }^{2}\right)\right]^{-1} b^{2} m^{2},
\end{aligned}
$$

with $\quad \alpha^{*}=\left(\rho_{1 x}{ }^{2}+\sigma_{\mu 1}{ }^{2} / \sigma_{x}^{2}\right)^{-1} \rho_{1 x}$,

$\sigma_{\pi}^{2}\left(\eta_{1}\right)=\left(\rho_{1 x}^{2}+\sigma_{\mu 1}{ }^{2} / \sigma_{x}^{2}\right)^{-1} \rho_{1 x}{ }^{2} \sigma_{x}^{2}$, and

$\mathrm{E}\left[y_{1} \mid \hat{a}, z, \eta_{1}\right]=\left(m^{2}+r \sigma_{\pi}^{2} / \alpha^{* 2}\right)^{-1} b m^{3}$. 
Table 2

Market Price and Price Sensitivity Under $\boldsymbol{\eta}_{1}$ and $\boldsymbol{\eta}_{2}$

$\eta_{1}$ - disclosure of $y_{1}$

$$
\begin{aligned}
& \pi\left(\eta_{1}\right)=\pi_{o}\left(\eta_{1}\right)+\left(\rho_{1 x}{ }^{2}+\sigma_{\mu 1}{ }^{2} / \sigma_{x}^{2}\right)^{-1} \rho_{1 x} m_{11} a_{1}+\left(\rho_{1 x}{ }^{2}+{\sigma_{\mu 1}}^{2} / \sigma_{x}^{2}\right)^{-1} \rho_{1 x} m_{12} a_{2}+\alpha^{*}\left(\rho_{1 x} \mathcal{E}_{x}+\mathcal{E}_{\mu 1}\right), \\
& \mu_{11} \equiv\left(\rho_{1 x}{ }^{2}+{\sigma_{\mu 1}}^{2} / \sigma_{x}{ }^{2}\right)^{-1} \rho_{1 x} m_{11}, \quad \text { and } \quad \mu_{12} \equiv\left(\rho_{1 x}{ }^{2}+\sigma_{\mu 1}{ }^{2} / \sigma_{x}^{2}\right)^{-1} \rho_{1 x} m_{12} .
\end{aligned}
$$

$\eta_{2}$ - disclosure of $y_{1}$ and $y_{2}$

$$
\begin{aligned}
& \pi\left(\eta_{2}\right)= \pi_{o}\left(\eta_{2}\right)+Q^{-1}\left[\left(\rho_{1 x} \sigma_{\mu 2}-\rho \rho_{2 x} \sigma_{\mu 1}\right) \sigma_{\mu 2} m_{11}+\left(\rho_{2 x} \sigma_{\mu 1}-\rho \rho_{1 x} \sigma_{\mu 2}\right) \sigma_{\mu 1} m_{21}\right] \sigma_{x}^{2} a_{1} \\
&+Q^{-1}\left[\left(\rho_{1 x} \sigma_{\mu 2}-\rho \rho_{2 x} \sigma_{\mu 1}\right) \sigma_{\mu 2} m_{12}+\left(\rho_{2 x} \sigma_{\mu 1}-\rho \rho_{1 x} \sigma_{\mu 2}\right) \sigma_{\mu 1} m_{22}\right] \sigma_{x}^{2} a_{2} \\
&+Q^{-1}\left[\rho_{1 x}^{2} \sigma_{\mu 2}^{2}-\rho \rho_{1 x} \rho_{2 x} \sigma_{\mu 1} \sigma_{\mu 2}+\rho_{2 x}^{2} \sigma_{\mu 1}^{2}-\rho \rho_{1 x} \rho_{2 x} \sigma_{\mu 1} \sigma_{\mu 2}\right] \sigma_{x}^{2} \varepsilon_{x} \\
&+Q^{-1} \sigma_{x}^{2}\left[\left(\rho_{1 x} \sigma_{\mu 2}-\rho \rho_{2 x} \sigma_{\mu 1}\right) \sigma_{\mu 2} \varepsilon_{\mu 1}+\left(\rho_{2 x} \sigma_{\mu 1}-\rho \rho_{1 x} \sigma_{\mu 2}\right) \sigma_{\mu 1} \mathcal{E}_{\mu 2}\right], \\
& \mu_{21} \equiv Q^{-1}\left[\left(\rho_{1 x} \sigma_{\mu 2}-\rho \rho_{2 x} \sigma_{\mu 1}\right) \sigma_{\mu 2} m_{11}+\left(\rho_{2 x} \sigma_{\mu 1}-\rho \rho_{1 x} \sigma_{\mu 2}\right) \sigma_{\mu 1} m_{21}\right] \sigma_{x}^{2}, \text { and } \\
& \mu_{22} \equiv Q^{-1}\left[\left(\rho_{1 x} \sigma_{\mu 2}-\rho \rho_{2 x} \sigma_{\mu 1}\right) \sigma_{\mu 2} m_{12}+\left(\rho_{2 x} \sigma_{\mu 1}-\rho \rho_{1 x} \sigma_{\mu 2}\right) \sigma_{\mu 1} m_{22}\right] \sigma_{x}^{2},
\end{aligned}
$$

with $Q \equiv\left(\rho_{1 x}{ }^{2} \sigma_{x}^{2}+{\sigma_{\mu 1}}^{2}\right)\left(\rho_{2 x}{ }^{2} \sigma_{x}^{2}+{\sigma_{\mu 2}}^{2}\right)-\left(\rho_{1 x} \rho_{2 x} \sigma_{x}^{2}+\rho \sigma_{\mu 1} \sigma_{\mu 2}\right)^{2}$. 
Figure 1

Value of a Publicly Reported Contractible Performance Measure $y_{c}$ Given the Disclosure of Two Non-contractible Performance Measures Under $\eta_{2}$

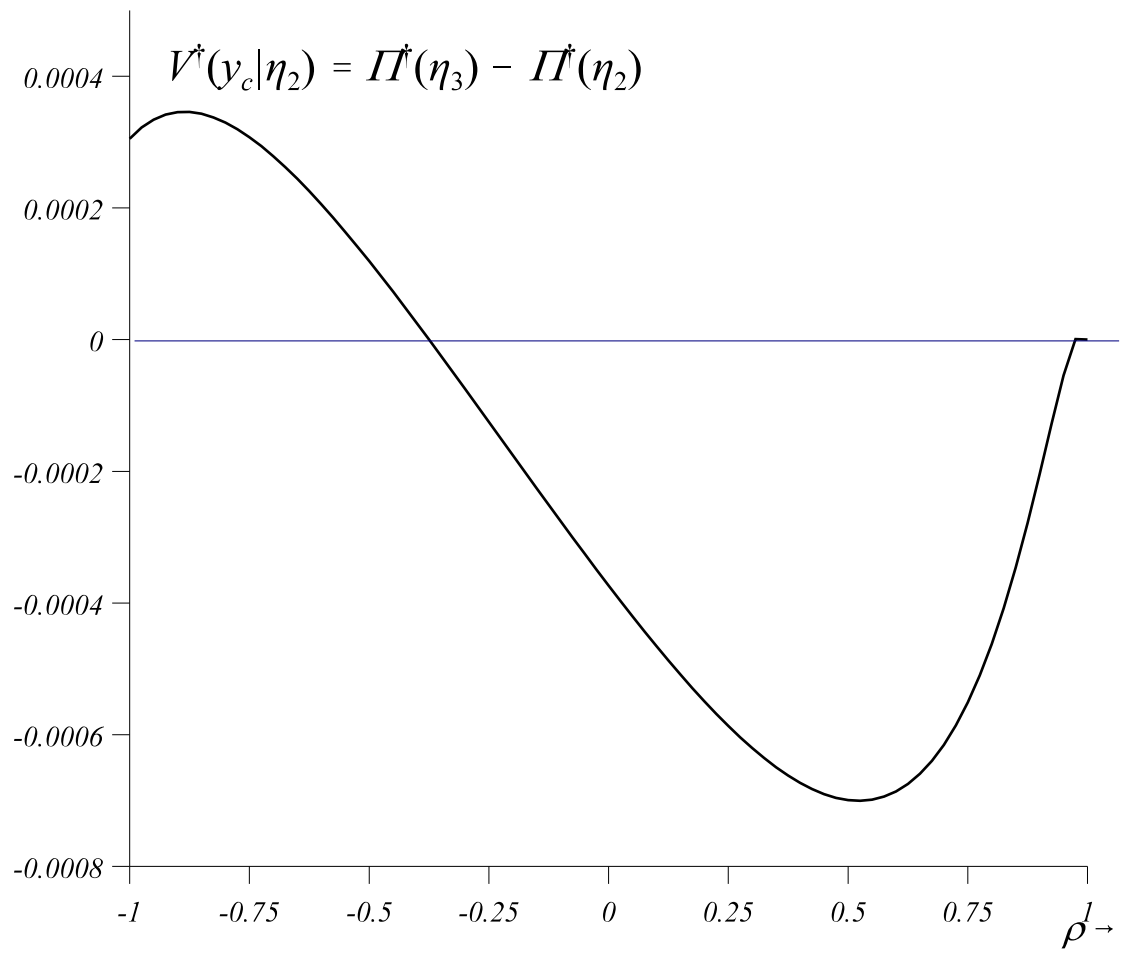

